\title{
Small regulatory RNA and Legionella pneumophila
}

\author{
Sébastien P. Faucher ${ }^{*}$ and Howard A. Shuman ${ }^{2}$ \\ Complex Traits Group, Department of Microbiology, McGill University, Montreal, QC, Canada \\ 2 Department of Microbiology, Cummings Life Sciences Center, University of Chicago, Chicago, IL, USA
}

\section{Edited by:}

Carmen Buchrieser, Pasteur Institute,

France

Reviewed by:

Michele S. Swanson, University of Michigan, USA

Jörg Vogel, University of Würzburg, Germany

Gisela Storz, National Institutes of Health, USA

\section{*Correspondance:}

Sébastien P. Faucher, Complex Traits Group, Department of Microbiology, McGill University, 3649 Sir-William-

Osler Promenade, Montreal, QC,

Canada H3G OB1.

e-mail: sebastien.faucher@gmail.com
Legionella pneumophila is a gram-negative bacterial species that is ubiquitous in almost any aqueous environment. It is the agent of Legionnaires' disease, an acute and often under-reported form of pneumonia. In mammals, L. pneumophila replicates inside macrophages within a modified vacuole. Many protein regulators have been identified that control virulence-related properties, including RpoS, LetA/LetS, and PmrA/PmrB. In the past few years, the importance of regulation of virulence factors by small regulatory RNA (sRNAs) has been increasingly appreciated. This is also the case in L. pneumophila where three sRNAs (RsmY, RsmZ, and 6S RNA) were recently shown to be important determinants of virulence regulation and 79 actively transcribed sRNAs were identified. In this review we describe current knowledge about sRNAs and their regulatory properties and how this relates to the known regulatory systems of $L$. pneumophila. We also provide a model for sRNA-mediated control of gene expression that serves as a framework for understanding the regulation of virulence-related properties of L. pneumophila.

\section{Keywords: CsrA, RsmY, RsmZ, 6S RNA, cyclic di-GMP, CRISPR}

\section{INTRODUCTION}

Legionella pneumophila is the causative agent of Legionnaires' disease, an acute form of pneumonia (Fraser et al., 1977). It is a common, but often underestimated, cause of community-acquired and nosocomial pneumonia. The case-fatality rate of Legionellosis ranges between 10 and $40 \%$ and may approach 50\% in nosocomial outbreaks, particularly among individuals with compromised health status (Benin et al., 2002). In Germany, where pneumonia causes are systematically investigated, Legionella is a leading cause of community-acquired pneumonia (von Baum et al., 2008).

Legionella pneumophila is commonly found in almost all natural and engineered water systems where it replicates in a variety of phagocytic protozoa, including Hartmannella vermiformis. Transmission mechanisms are still unclear, but a clear association was found between local watershed hydrology and Legionellosis risk in Toronto (Ng et al., 2008), which indicates that environmental factors are key players in transmission to humans. In people, infection is thought to occur by inhalation of contaminated water droplets.

Once in the lungs, L. pneumophila infects and replicates inside alveolar macrophages. To successfully infect and grow inside host cells, L. pneumophila circumvents normal endocytic trafficking pathways and inhibits phagosome acidification and fusion with lysosomes to establish a permissive replication niche called the Legionella containing vacuole (LCV) (Franco et al., 2009). The LCV is characterized by recruitment of early secretory vesicles, mitochondria, and membrane vesicles derived from the Golgi and endoplasmic reticulum (Roy and Tilney, 2002; Molofsky and Swanson, 2004; Shin and Roy, 2008). Central to the formation of the LCV and intracellular growth is the $\mathrm{Icm} / \mathrm{Dot}$ type IVB secretion system, which translocates approximately 200 diverse effector proteins to the cytosol and LCV membrane (Segal and Shuman, 1998; Segal et al., 1998; Vogel et al., 1998; Cazalet et al., 2004; Chien et al., 2004; de Felipe et al., 2005; Burstein et al., 2009; Hubber and Roy, 2010; Faucher et al., 2011; Huang et al., 2011; Zhu et al., 2011).
Because L. pneumophila has evolved in a variety of niches, including aquatic environments, biofilms as well as within diverse hosts, different stress response pathways and virulence pathways must be correctly regulated. Although little is known about gene regulation in natural or engineered aquatic environments, several two-component systems are known to be involved in the regulation of stress response pathways and virulence factors required during host cells infection. These include PmrA/PmrB (Zusman et al., 2007), CpxR/CpxA (Altman and Segal, 2008) and LetA/LetS (Hammer et al., 2002). In addition, the sigma factor $\operatorname{RpoS}\left(\sigma^{S}\right)$ has been shown to regulate a number of known virulence factors including many $\mathrm{Icm} / \mathrm{Dot}$ effectors (Hovel-Miner et al., 2009) and is required for intracellular multiplication in ameba and primary macrophages but not in macrophage-like cell lines, probably because of their reduced antimicrobial capacity (Hales and Shuman, 1999; AbuZant et al., 2006).

There is an increased awareness of the role of small regulatory RNAs (sRNAs) in the regulation of virulence factors and other processes in bacterial pathogens (Papenfort and Vogel, 2010). sRNAs are short (40-500 nt) RNA molecules that typically do not encode proteins and mainly perform regulatory functions. They can originate from either primary transcripts, meaning the sRNA is transcribed from its own promoter and its transcription stops at a Rho-independent terminator, or from the processing of larger transcripts. The vast majority of sRNAs are post-transcriptional regulators that can either inhibit or enhance mRNA translation of the target mRNAs (Waters and Storz, 2009). Other sRNAs regulate gene expression by binding to and interfering with regulatory proteins and have global effects on gene expression. Riboswitches and untranslated regions (UTR) are not sRNA per se, being an intrinsic part of the mRNA, but they are often found by the methodologies used to identify small RNA molecules. 
Putative sRNA molecules expressed by L. pneumophila were identified by both a bioinformatic approach as well as by deep RNAsequencing from growth in broth and inside A. castellanii (Faucher et al., 2010; Weissenmayer et al., 2011). In addition, a number of sRNAs have been implicated in the regulation of virulence factors of L. pneumophila, including the CsrB homologs RsmY and RsmZ (Rasis and Segal, 2009a; Sahr et al., 2009) and the RNA polymerase (RNAP) regulator 6S RNA (Faucher et al., 2010). This review aims to describe the current knowledge about sRNAs in general and provide a global perspective of the involvement of sRNA regulation systems in the behavior of L. pneumophila.

\section{BASE-PAIRING sRNAs}

The most common type of regulatory sRNA are base-pairing sRNAs. They are short, highly structured RNA molecules that are complementary to some degree to their target mRNAs and are therefore often called antisense sRNAs (Brantl, 2007). Base-pairing sRNAs can have a positive or a negative effect on expression of the target gene. Binding of the sRNA at or near the ribosomal binding site (RBS) prevents recognition by the ribosome and subsequent translation (Figures 1B,C). Alternatively, binding of the sRNA could change the secondary structure of the mRNA and free the RBS to permit translation initiation (Figure 1C). sRNA-binding to the mRNA can also induce its degradation by recruiting RNases (Waters and Storz, 2009). Base-pairing sRNAs can be encoded in cis or in trans.

\section{CIS-ENCODED BASE-PAIRING sRNAs}

Cis-encoded sRNAs are antisense RNA molecules encoded on the complementary strand of their target RNA gene (Figure 1B). Therefore, they share extensive sequence complementarity with the target mRNA but do not necessarily form long RNA duplexes (Brantl, 2007). Thirty-three sRNAs were recently identified in L. pneumophila that were at least partially complementary to genes encoding protein, some being known virulence factors (Weissenmayer et al., 2011; Table 1). Lpr0020 is encoded antisense to lpg0644, which encodes a homolog of RtxA involved in intracellular survival and modification of trafficking (Cirillo et al., 2001, 2002). Another sRNA, Lpr0050, is found antisense to the Icm/Dot effector SdeA ( $l p g 2157$; Bardill et al., 2005). Two sRNAs, Lpr0003 and Lpr0004, are antisense to the gene encoding the Icm/ Dot effector LegA10, and are expressed during intracellular growth in A. castellanii.

Lpr0018 is encoded antisense to comEC (also known as comA, lpg0626) and would form a duplex with the $5^{\prime}$ end of the coding sequence and partially with a putative $5^{\prime} \mathrm{UTR}$. ComEC is predicted to

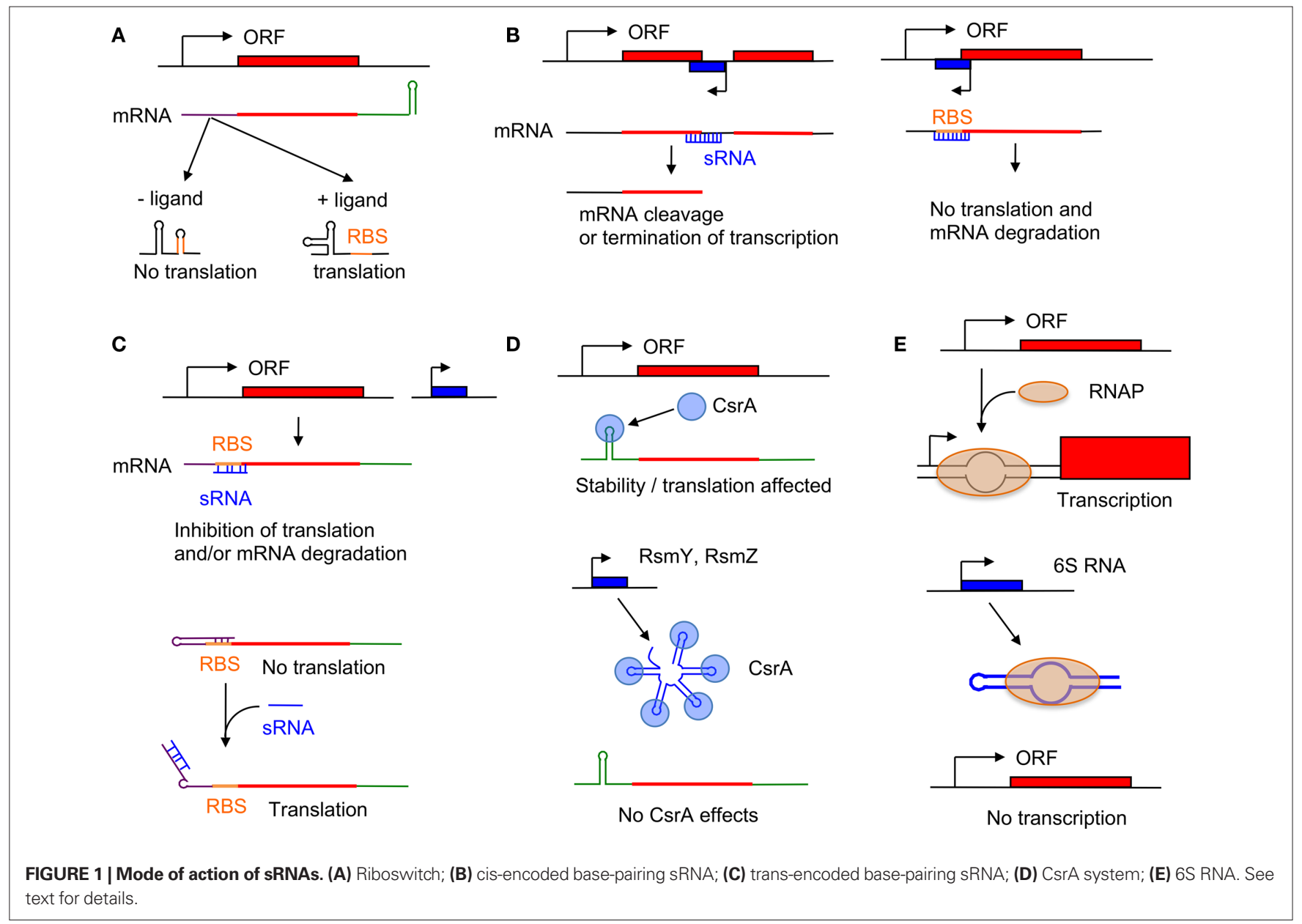


Table 1 | Small RNA molecules identified in L. pneumophila.

\begin{tabular}{|c|c|c|c|c|c|c|c|}
\hline Name & $5^{\prime}$ end $^{1}$ & $3^{\prime}$ end $^{1}$ & Size (nt) & Regulator & Target $^{5}$ & Note & Reference \\
\hline \multicolumn{8}{|c|}{ PROTEIN-BINDING sRNAs } \\
\hline RsmY & 7168 & $7059^{2}$ & $110^{2}$ & LetA, RpoS & CsrA & $\begin{array}{l}\triangle r s m Y Z \text { is defective for } \\
\text { intracellular multiplication }\end{array}$ & $\begin{array}{l}\text { Rasis and Segal (2009a), } \\
\text { Sahr et al. (2009), }\end{array}$ \\
\hline RsmZ & 1892720 & 1892592 & 132 & LetA, RpoS & CsrA & & Hovel-Miner et al. (2009) \\
\hline 6S RNA & 951819 & $\begin{array}{l}951673 \\
951638\end{array}$ & 147,182 & & RNAP & $\begin{array}{l}\text { Required for optimal } \\
\text { intracellular multiplication }\end{array}$ & Faucher et al. (2010) \\
\hline $\begin{array}{l}\text { 6S2 RNA } \\
\text { antisense }\end{array}$ & 80859 & 81037 & 178 & & & & Weissenmayer et al. (2011) \\
\hline \multicolumn{8}{|c|}{ CIS-ENCODED BASE-PAIRING sRNAs } \\
\hline |pr0002 & 33166 & 33516 & 350 & & lpg0027 & & Weissenmayer et al. (2011) \\
\hline Ipr0003 & 45316 & 45539 & 223 & & Ipg0038 & & Weissenmayer et al. (2011) \\
\hline Ipr0012 & 369510 & 369457 & 53 & & Ipg0320 & & Weissenmayer et al. (2011) \\
\hline Ipr0015 & 425773 & 425602 & 171 & & lpg0384 & & Weissenmayer et al. (2011) \\
\hline Ipr0016 & 532291 & 532155 & 136 & & lpg0494 & & Weissenmayer et al. (2011) \\
\hline Ipr0017 & 539616 & 539866 & 250 & & Ipg0499, Ipg0500 & & Weissenmayer et al. (2011) \\
\hline Ipr0018 & 662203 & 662439 & 236 & & Ipg0626 & & Weissenmayer et al. (2011) \\
\hline Ipr0019 & 662451 & 663193 & 742 & & Ipg0627, Ipg0628 & & Weissenmayer et al. (2011) \\
\hline Ipr0020 & 686010 & 685864 & 146 & & lpg0644 & & Weissenmayer et al. (2011) \\
\hline Ipr0021 & 744637 & 744929 & 292 & & Ipg0691 & & Weissenmayer et al. (2011) \\
\hline Ipr0025 & 825408 & 825265 & 143 & & lpg0754 & & Weissenmayer et al. (2011) \\
\hline Ipr0026 & 837669 & 837871 & 202 & & lpg0766 & & Weissenmayer et al. (2011) \\
\hline Ipr0028 & 871501 & 871409 & 92 & & lpg0796 & & Weissenmayer et al. (2011) \\
\hline Ipr0049 & 2389440 & 2389117 & 323 & & lpg2142 & & Weissenmayer et al. (2011) \\
\hline Ipr0050 & 2418574 & 2418506 & 68 & & lpg2157 & & Weissenmayer et al. (2011) \\
\hline Ipr0053 & 2564155 & 2564056 & 99 & & $\operatorname{lpg} 2261$ & & Weissenmayer et al. (2011) \\
\hline Ipr0054 & 2574935 & 2574715 & 220 & & lpg2271 & & Weissenmayer et al. (2011) \\
\hline Ipr0058 & 2867524 & 2867415 & 109 & & lpg2535, Ipg2536 & & Weissenmayer et al. (2011) \\
\hline Ipr0062 & 2948692 & 2948783 & 91 & & $\operatorname{lpg} 2612$ & & Weissenmayer et al. (2011) \\
\hline Ipr0065 & 3095189 & 3095277 & 88 & & $\operatorname{lpg} 2744$ & & Weissenmayer et al. (2011) \\
\hline Ipr0071 & 3351555 & 3351860 & 305 & & $\operatorname{lpg} 2961$ & & Weissenmayer et al. (2011) \\
\hline Ipr0072 & 3374990 & 3375092 & 102 & & $\operatorname{lpg} 2981$ & & Weissenmayer et al. (2011) \\
\hline \multicolumn{8}{|c|}{ TRANS-ENCODED PUTATIVE BASE-PAIRING sRNAs } \\
\hline LprA & 2013775 & 2013510 & 265 & RpoS, OxyR & & Correspond to Lpr0041 & $\begin{array}{l}\text { Faucher et al. (2010), } \\
\text { Weissenmayer et al. (2011) }\end{array}$ \\
\hline LprB & 2022555 & 2022672 & 117 & OxyR & & & Faucher et al. (2010) \\
\hline LprC & 978559 & 978676 & 117 & & & & Faucher et al. (2010) \\
\hline LprD & 3321618 & 3321516 & 103 & & & & Faucher et al. (2010) \\
\hline LprE & 33394003 & 33393503 & $<50^{3}$ & & & & Faucher et al. (2010) \\
\hline |pr0001 & 18080 & 18214 & 134 & & & Functional $^{6}$ & Weissenmayer et al. (2011) \\
\hline Ipr0005 & 51416 & 51182 & 234 & & & & Weissenmayer et al. (2011) \\
\hline Ipr0007 & 262199 & 262033 & 166 & & & Functional $^{6}$ & Weissenmayer et al. (2011) \\
\hline Ipr0010 & 341341 & 341434 & 93 & & & Very unstable & Weissenmayer et al. (2011) \\
\hline
\end{tabular}


Table 1 | Continued

\begin{tabular}{|c|c|c|c|c|c|c|c|}
\hline Name & $5^{\prime}$ end ${ }^{1}$ & $3^{\prime}$ end $^{1}$ & Size (nt) & Regulator & Target & Note & Reference \\
\hline Ipr0011 & 360467 & 360391 & 76 & & & & Weissenmayer et al. (2011) \\
\hline Ipr0013 & 411825 & 412167 & 342 & & & & Weissenmayer et al. (2011) \\
\hline Ipr0022 & 753291 & 753083 & 208 & & & & Weissenmayer et al. (2011) \\
\hline Ipr0023 & 753084 & 753379 & 295 & & & Functional ${ }^{6}$ & Weissenmayer et al. (2011) \\
\hline Ipr0024 & 816705 & 816590 & 115 & & & Functional ${ }^{6}$ & Weissenmayer et al. (2011) \\
\hline Ipr0030 & 1102961 & 1103162 & 201 & & & & Weissenmayer et al. (2011) \\
\hline Ipr0032 & 1215340 & 1215182 & 158 & & & Functional ${ }^{6}$ & Weissenmayer et al. (2011) \\
\hline Ipr0034 & 1333886 & 1334233 & 347 & & & Functional ${ }^{6}$ & Weissenmayer et al. (2011) \\
\hline Ipr0035 & 1355695 & 1355444 & 251 & & & & Weissenmayer et al. (2011) \\
\hline Ipr0038 & 1444737 & 1444509 & 228 & & & & Weissenmayer et al. (2011) \\
\hline Ipr0039 & 1869948 & 1869698 & 250 & & & & Weissenmayer et al. (2011) \\
\hline Ipr0047 & 2358694 & 2358599 & 95 & & & & Weissenmayer et al. (2011) \\
\hline Ipr0048 & 2360881 & 2360970 & 89 & & & Functional ${ }^{6}$ & Weissenmayer et al. (2011) \\
\hline Ipr0051 & 2432864 & 2432952 & 88 & & & & Weissenmayer et al. (2011) \\
\hline Ipr0052 & 2549075 & 2548822 & 253 & & & & Weissenmayer et al. (2011) \\
\hline Ipr0055 & 2769045 & 2768903 & 142 & & & & Weissenmayer et al. (2011) \\
\hline Ipr0056 & 2768934 & 2769061 & 127 & & & & Weissenmayer et al. (2011) \\
\hline Ipr0057 & 2862083 & 2862349 & 266 & & & & Weissenmayer et al. (2011) \\
\hline Ipr0059 & 2877260 & 2877374 & 114 & & & & Weissenmayer et al. (2011) \\
\hline Ipro060 & 2921311 & 2921667 & 356 & & & Functional ${ }^{6}$ & Weissenmayer et al. (2011) \\
\hline Ipr0061 & 2921870 & 2921081 & 789 & & & & Weissenmayer et al. (2011) \\
\hline Ipr0063 & 2981537 & 2981411 & 126 & & & & Weissenmayer et al. (2011) \\
\hline $\begin{array}{l}\text { lpg0165 } \\
-3 \text { UTR }\end{array}$ & $195661^{3}$ & $195572^{3}$ & 89 & & & & Faucher et al. (2010) \\
\hline infA-3'UTR & $1976419^{3}$ & $1976584^{3}$ & 165 & & & & Faucher et al. (2010) \\
\hline gltX-3'UTR & $2132087^{3}$ & $2131915^{3}$ & 172 & & & & Faucher et al. (2010) \\
\hline rpsU-3'UTR ${ }^{4}$ & $2663501^{3}$ & $2663567^{3}$ & 66 & & & & Faucher et al. (2010) \\
\hline$r p s U-3^{\prime} U T R^{4}$ & $2663501^{3}$ & $2663681^{3}$ & 180 & & & & Faucher et al. (2010) \\
\hline $\begin{array}{l}\text { lpg2505 } \\
-3^{\prime} U T R\end{array}$ & $2824648^{3}$ & $2824828^{3}$ & 180 & & & & Faucher et al. (2010) \\
\hline \multicolumn{8}{|l|}{ OTHER } \\
\hline tmRNA & 172820 & 173374 & 554 & & & & Weissenmayer et al. (2011) \\
\hline Rnase $\mathrm{P}$ & 1944961 & 1944585 & 375 & & & & Weissenmayer et al. (2011) \\
\hline
\end{tabular}

'The position of the sRNA is given relative to L. pneumophila Philadelphia-1 genome.

${ }^{2}$ The 3 ' end and the size given for RsmY are based on published results in L. pneumophila Paris. In L. pneumophila Philadelphia-1, the 3' end of RsmY is at position 7090 for a size of $79 \mathrm{nt}$, as determined by $3^{\prime}$ RACE.

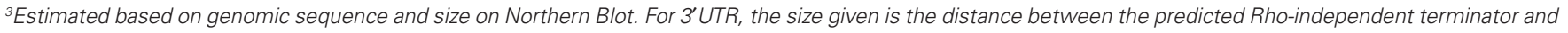
the stop codon of the upstream gene. The size of all 3'UTR was higher than $500 \mathrm{nt}$ as observed on northern blot.

${ }^{4}$ Two overlapping putative sRNAs were predicted in this region with distinc predicted terminators.

${ }^{5}$ For cis-encoded sRNAs, the target correspond to the gene on the complementary strand of the sRNA.

${ }^{6}$ Trans-encoded sRNAs identified by Weissenmayer et al. (2011) were predicted as functional if the predicted structure was found to be stable. 
be part of the machinery involved in DNA uptake in L. pneumophila. Competence for natural transformation is induced by treatment that triggers stalling of the replication fork, such as UV irradiation and exposure to bicyclomycin (Charpentier et al., 2011). Some evidence previously suggested that sRNA could be involved in regulation of competence in L. pneumophila. First, deletion of the rnr gene, encoding RNase R, was found to induce competence and resulted in the accumulation of small RNA molecules originating from highly structured 16S rRNA and tmRNA (see below; Charpentier et al., 2008). Whether or not these two phenotypes are related requires clarification. Second, the Escherichia coli homolog of the L. pneumophila competence repressor ProQ (Sexton and Vogel, 2004) was found to work as a RNA chaperone to allow translation of proP mRNA, involved in the uptake of osmoprotectants (Chaulk et al., 2011). Taken together, these facts could lead one to hypothesize a regulatory model in which ProQ is essential to inhibit degradation, by RNase R, of the sRNA Lpr0018, which would mediate degradation of comEC mRNA, similar to the mechanism depicted in Figure 1B. Therefore, in the absence of ProQ or RNase R, comEC would be stabilized and efficiently translated. Alternatively, the sRNA Lpr0018 could stabilize comEC mRNA, allowing its transcription, while ProQ could act as a negative regulator of Lpr0018, potentially by targeting it for degradation. However, to our knowledge, such a mechanism has yet to be described for cis-encoded sRNA. Another sRNA, Lpr0019, is $742 \mathrm{nt}$ long and is complementary to the $5^{\prime}$ end of $\operatorname{lpg} 0627$ and to the $3^{\prime}$ end of $\operatorname{lpg} 0628$. Both genes are part of a predicted polycistronic RNA composed of lpg0632-lpg0627 encoding subunits of the type IV pili, which was associated with competence (Stone and Kwaik, 1999). Lpr0019 could possibly be involved in induction of competence in a manner similar to what we suggested for Lpr0018. Of course, those hypotheses will need to be tested experimentally. Nonetheless, the finding that two sRNAs are encoded antisense to key players of DNA uptake by L. pneumophila strongly suggest that its induction is regulated at the post-transcriptional level. Recently, induction of competence in Vibrio cholerae was found to be dependent on the expression of a trans-encoded sRNA (TfoR), which allows translation of the positive regulator TfoX (Yamamoto et al., 2011).

Lpr0036 is encoded antisense to $\operatorname{lvr}$ ( $\operatorname{lpg} 1259)$, the first gene of the $l v r / l v h$ locus encoding a Type IVA secretion system, involved in conjugation (Segal et al., 1999). However, the role of LvrA is currently unknown and it is difficult at this point to speculate a possible role for this sRNA.

\section{TRANS-ENCODED BASE-PAIRING sRNAs}

In contrast to cis-encoded sRNA, trans-encoded base-pairing sRNAs are not physically linked to their mRNA target and the formation of RNA duplexes are mediated by short imperfect RNA interactions (Figure 1C). The function of many of the trans-encoded basepairing sRNAs depends on the RNA-binding protein Hfq, which is thought to enhance the likelihood of a productive interaction between the sRNA and its target (Waters and Storz, 2009). This is in contrast to cis-encoded base-pairing sRNA that do not generally require the participation of a RNA chaperone (e.g., Hfq) to bind their target mRNA (Brantl, 2007).

In bacterial pathogens, deletion of the $h f q$ gene often leads to a reduction in virulence, as was observed for E. coli, Salmonella, Shigella, Yersinia, and Listeria (Reviewed in Chao and Vogel, 2010).
For example, in E. coli, Hfq was shown to regulate the locus of enterocytes effacement (LEE) encoding a type III secretion system (TTSS; Hansen and Kaper, 2009; Shakhnovich et al., 2009). In the intracellular pathogen Salmonella enterica serovar Typhimurium, $\mathrm{Hfq}$ is necessary for optimal growth in epithelial cells and macrophages (Sittka et al., 2007). Burkholderia cenocepacia encodes two Hfq homologs and both of them are required for optimal resistance to stress and virulence (Ramos et al., 2011). Deletion of the hfq gene of Staphylococcus aureus has no effect on metabolism but reduces virulence (Bohn et al., 2007; Liu et al., 2010). However, in Neisseria gonorrhoeae, deletion of $h f q$ leads to only a weak reduction of virulence (Dietrich et al., 2009). Moreover, in some bacteria, $\mathrm{Hfq}$ is required for the function of some sRNAs but dispensable for others. For example, in V. cholerae, Hfq is required for the control of the quorum sensing systems by the sRNAs Qrr1-Qrr4, but dispensable for the repression of ompA by VrrA (Lenz et al., 2004; Song et al., 2008). It is noteworthy that Helicobacter pylori does not encode an Hfq homolog but still expresses hundreds of sRNAs (Sharma et al., 2010). This suggests that in some bacterial species, the function mediated by Hfq is not necessary for sRNA-mediated gene regulation or that an as yet unknown protein could carry out a similar function. Following genome-wide identification of Hfqbinding sRNAs, it was postulated that even in E. coli, some basepairing sRNAs might not bind to, or use Hfq (Zhang et al., 2003). Careful review of the Hfq-related literature lead Jousselin et al. (2009) to postulate that the need for Hfq in mRNA-sRNA interaction is related to a number of factors. First, the higher the overall GC content of the bacterial genome the more likely Hfq is required and $\mathrm{Hfq}$ seems to be dispensable in bacteria whose genomes display a low GC value, such as S. aureus (32\% GC). Second, Hfq is dispensable when the sRNA-mRNA interaction is mediated by long $(>30)$ and uninterrupted pairing. Third, they observed a correlation between a requirement for $\mathrm{Hfq}$ and the $\mathrm{C}$-terminal extension length of $\mathrm{Hfq}$, which forms an mRNA interaction surface. Hfq proteins that have a short $\mathrm{C}$-terminus tend to be found in bacteria in which Hfq is dispensable.

In L. pneumophila, deletion of the $h f q$ gene affects the duration of the lag phase after inoculation in fresh broth (McNealy et al., 2005). Moreover, the L. pneumophila $h f q$ mutant shows a reduced growth rate in chemically defined medium containing low concentrations of iron and a reduction in the expression of the ferric uptake regulator (fur). In E. coli, the RyhB sRNA negatively regulates expression of fur in a Hfq-dependant manner (Vecerek et al., 2007). In addition, the L. pneumophila $h f q$ mutant shows a small reduction in intracellular growth (McNealy et al., 2005). The somewhat limited effect of deleting the $h f q$ gene on L. pneumophila phenotypes suggests that Hfq is not critical for sRNA-mRNA interactions in this organism. The GC content of the L. pneumophila genome is low $(38 \%)$ and alignment of its $\mathrm{Hfq}$ protein sequence with other homologs (Figure 2) reveals that the C-terminal region is short and comparable to the length of the $V$. cholerae $\mathrm{Hfq}$ that is not essential for all mRNA-sRNA interactions. According to the postulates of Jousselin et al. (2009), one could hypothesize that Hfq will not be required for all sRNA-mRNA interactions in L. pneumophila.

Nonetheless, one can speculate that in L. pneumophila, basepairing sRNAs acting through $\mathrm{Hfq}$ may regulate iron acquisition, virulence-related functions and possibly other systems as well, 


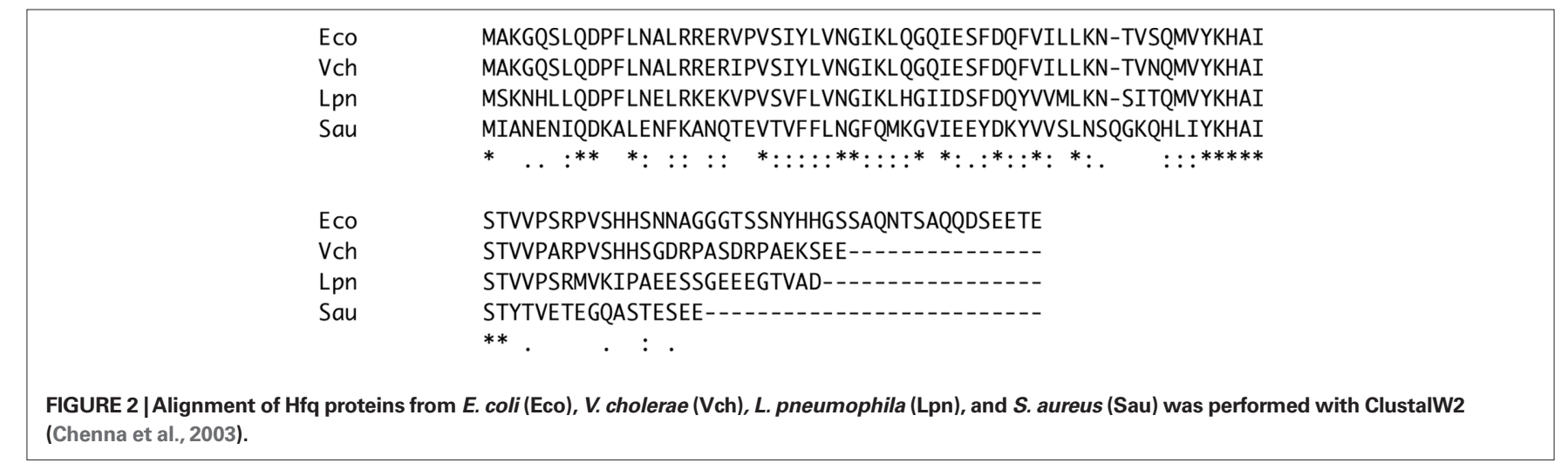

although Hfq function would not be essential for these. Expression profiling of a $h f q$-deficient L. pneumophila strain would shed light on the importance of $\mathrm{Hfq}$ on gene regulation and be of great help at identifying phenotypes that could be affected by it. A similar approach was used for other bacteria such as E. coli (Zhang et al., 2003), Typhimurium (Sittka et al., 2008), B. cenocepacia (Ramos et al., 2011), Pseudomonas aeruginosa (Sonnleitner et al., 2006), and $N$. gonorrhoeae (Dietrich et al., 2009). In addition, immunoprecipitation of Hfq with subsequent identification of bound sRNAs by enzymatic RNA-sequencing (Christiansen et al., 2006), tiling microarray (Zhang et al., 2003), or deep-sequencing (Sittka et al., 2008) would shed light on the mRNA species affected by Hfq and on the potential sRNAs whose functions are at least partially dependant on Hfq. Windbichler et al. (2008) have used an affinity chromatography procedure to identify RNA-binding proteins in E. coli. Briefly, they tagged a number of known sRNAs with a streptomycin-binding RNA aptamer, allowing them to bind to a streptomycin-coated column, which was then used to capture RNA-binding proteins from cellular extracts. They found that three proteins were consistently bound to a variety of sRNA sequences: Hfq, RNAP $\beta$-subunit and the small ribosomal subunit S1. Moreover, they showed that specific proteins could interact with a specific sRNA, depending on its sequence and secondary structure. Therefore, a hunt for sRNAbinding proteins is necessary to complete the sRNA-mediated regulatory landscape and to fully understand the extent of their impact on regulation of cellular functions.

In L. pneumophila, a number of trans-encoded base-pairing sRNA candidates have been identified but mechanistic studies are needed to evaluate their mode of action and to validate them as authentic base-pairing sRNAs (Table 1). Five intergenic RNAs were identified based on computer prediction by using the sRNA Predict software (Faucher et al., 2010). By searching for Rho-independent terminators in intergenic regions preceded by a sequence conserved in other L. pneumophila strains, 143 sRNA molecules were predicted. Using a custom-made microarray, the expression of 101 of these predicted sRNAs was monitored during growth in a variety of conditions. This two-step approach led to the identification of 12 sRNA molecules that were actively expressed, including $6 \mathrm{~S}$ RNA, six 3'UTR, and five sRNAs that are independently transcribed (Faucher et al., 2010; Table 1). At this point the functions of the five identified sRNAs are unclear. Interestingly, expression of LprA during exponential growth is dependant on OxyR but dependant on RpoS during post-exponential phase (Figure 3). Since RpoS is an important regulator of virulence, it is tempting to speculate that LprA could be part of its regulatory cascade and plays a role in expression of virulence factors. Regardless of the growth phase, the presence of $\mathrm{H}_{2} \mathrm{O}_{2}$ induces its expression, which suggests that LprA responds to oxidative stress. This is similar to the E. colisRNA OxyS, which is part of the oxidative stress response and reduces its mutagenic effects (Altuvia et al., 1997).

RNA-sequencing identified 38 sRNA molecules encoded in intergenic regions that could be considered as potential transencoded sRNAs (Weissenmayer et al., 2011; Table 1). Of these, nine were predicted to be functional based on the stability of their predicted secondary structures at $37^{\circ} \mathrm{C}$. The predicted structure of one sRNA (Lpr0010) was less stable than 1000 randomly permutated sequences of the same length and base composition at 20 or $37^{\circ} \mathrm{C}$, suggesting that it is under evolutionary pressure to form an unstable secondary structure. The biological relevance of this was not explored further, but one can hypothesize that the structure is only stable at low temperatures (less than $20^{\circ} \mathrm{C}$ ) and that it could be part of a cellular response to low temperature. Interestingly five sRNA pairs were identified, for which two distinct sRNA are transcribed antisense to each other (Weissenmayer et al., 2011). In E. coli, the sRNAs RyeB and SraC are encoded opposite to each other and RyeB is completely complementary to the longer SraC segment (Vogel et al., 2003). The size of $\mathrm{SraC}$ is $\approx 270 \mathrm{nt}$, but when RyeB is present a shorter band $(\approx 150 \mathrm{nt})$ is also detected. This reduction in size seems to be dependent on RNase III, suggesting that RyeB mediates degradation of SraC. For the sRNA pairs identified in Legionella, one sRNA can act as a negative regulator of the other, efficiently sequestering it by extended base-pairing and potentially targeting it for degradation. Moreover, mRNA can also regulates sRNAs. This mechanism, named trap-RNA, was described for the MicM sRNA that induces degradation of the YbfM porin mRNA. The $c h b$ polycistronic mRNA contain a sequence complementary to MicM and expression of the chb operon leads to MicM hybridization and degradation, resulting in stabilization of the $y b f M$ mRNA (Figueroa-Bossi et al., 2009; Overgaard et al., 2009). Again, additional work is needed to understand the regulatory functions of Legionella trans-encoded base-pairing sRNA.

There are a number of base-pairing sRNAs encoded in other bacterial genomes that are known to affect virulence. A few examples are provided below that might be relevant in the context of 


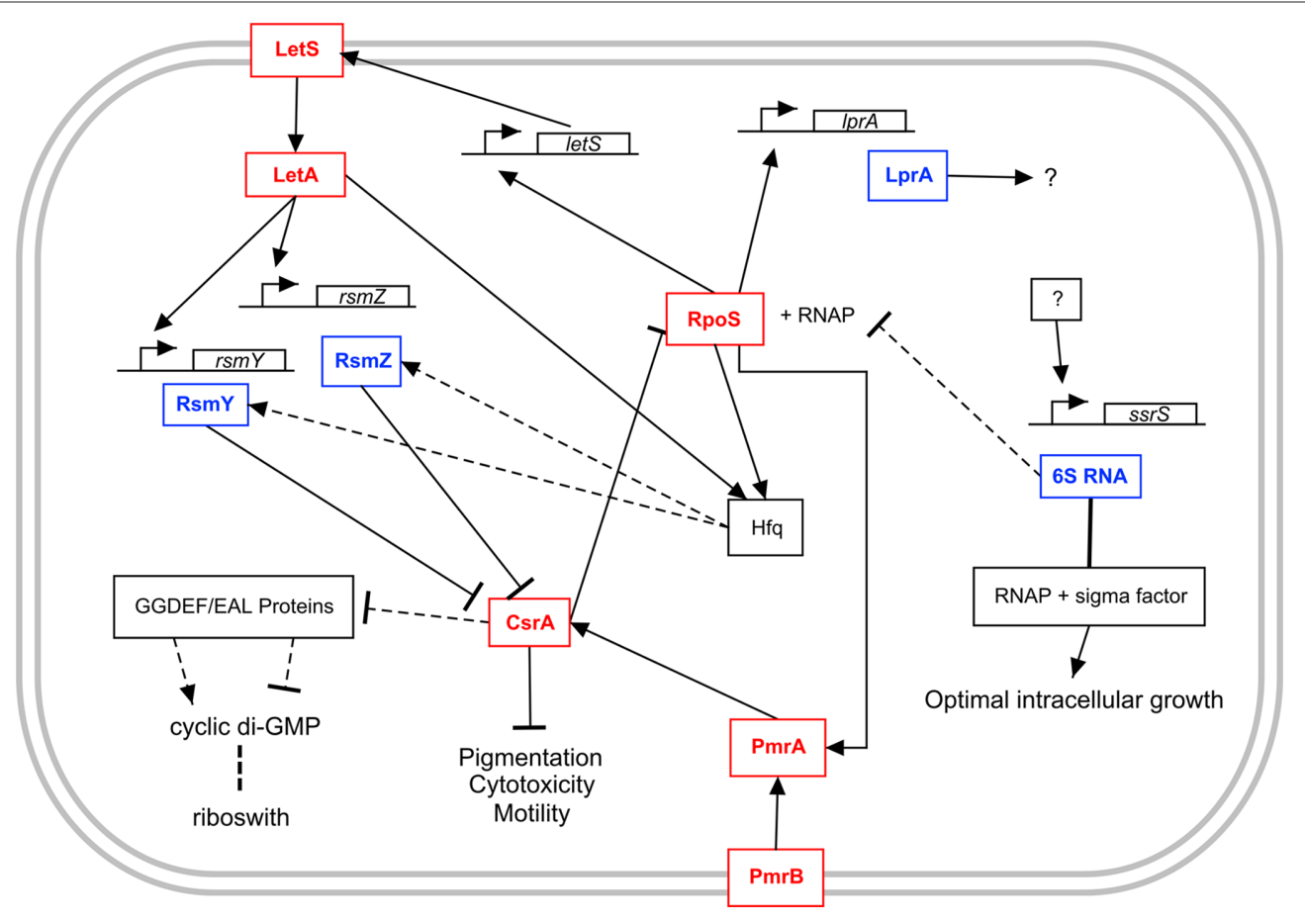

FIGURE 3 | Model of the regulatory networks involving sRNAs in $L$. pneumophila. The lines show interaction between the players: Arrow, activation; T bar, repression; dotted line, putative, or predicted interaction. See text for details.

L. pneumophila intracellular growth. One intracellular pathogen for which extensive identification and characterization of sRNA have been and are being performed is Salmonella. In this species, outer membrane protein (OMP) expression is regulated by a network of sRNAs. One of them, InvR, is encoded on the Salmonella pathogenicity island-1, acquired by horizontal gene transfer (HGT) and encoding the TTSS responsible for enterocyte invasion (Pfeiffer et al., 2007). Expression of this sRNA is dependant on HilD, a key regulator of TTSS expression. When the TTSS is expressed, InvR acts as a negative regulator of OmpD synthesis, one of the most abundant OMP in Typhimurium. Indirect evidence suggests that repression of OmpD could stabilize the membrane in the context of TTSS expression, allowing succesful translocation of bacterial effectors (Vogel, 2009). Therefore, InvR is thought to have helped establishment of the TTSS sequences after HGT by repressing expression of OMP that were incompatible with the virulence advantage provided by the TTSS (Vogel, 2009). Therefore, it is tempting to speculate that similar mechanisms exist in $L$. pneumophila to repress OMPs during expression of the $\mathrm{Icm} /$ Dot system, the Type IVA secretion system $(l v r / l v h)$ or the Tra conjugative system. However, to date, no trans-encoded sRNAs have been identified in the vicinity of these systems, but, as described above, one cis-encoded sRNA is antisense to lvrA (lpg1259).

The sRNA VrrA of $V$. cholerae is part of the membrane stress response pathway mediated by $\sigma^{\mathrm{E}}$ and targets ompA mRNA, presumably to limit synthesis of OMPs (Song et al., 2008). Deletion of $v r r A$ leads to an increase in the synthesis of outer membrane vesicles that are known to be involved in delivery of virulence factors to host cells (Mashburn-Warren and Whiteley, 2006). Moreover,
VrrA seems to negatively regulate expression of the adhesion molecule Tcp and therefore affects intestinal colonization (Song et al., 2008). There is structural similarity between VrrA and LprD of L. pneumophila and it is tempting to speculate a role for LprD in the regulation of OMP synthesis. However, structure comparisons of trans-encoded sRNAs have been of limited help for predicting function or targets, and an experimental strategy should be taken to determine if LprD regulates OMP synthesis.

The quorum system of $V$. cholerae comprises four redundant sRNAs named Qrr1-Qrr4 and two signaling molecules, the furanosyl borate diester (AI-2) and the $\alpha$-hydroxyketone Cqs (Lenz et al., 2004). At low cell density, the system positively regulates expression of Qrr1-Qrr4, which destabilize the mRNA of $h a p R$, a negative regulator of virulence. Therefore, at low cell density, hapR is degraded allowing expression of virulence traits. L. pneumophila also possesses a putative quorum system, based solely on the presence of the $\alpha$-hydroxyketone Lqs and the LqsR/LqsS two-components system (TCS) (Tiaden et al., 2007; Spirig et al., 2008). Beside the absence of AI-2 signaling in L. pneumophila the quorum system architecture of $L$. pneumophila and $V$. cholerae are quite similar (Tiaden et al., 2010). However, in L. pneumophila no sRNA has been implicated in this regulatory system as yet. Following RNA-sequencing, two sRNAs (Lpr0001, and Lpr0069) were found to have substantial homology both at the sequence and the secondary structure levels, which is reminiscent of the Qrr1-Qrr4 sRNAs (Weissenmayer et al., 2011). A search for homologous sequences throughout the genome revealed 20 more copies of these sRNAs, one (Lpr0049) being partially antisense to $l p g 2142$, which encodes a putative ORF. The consensus structure of these sRNAs is a long stem-loop with 
two central bulges comprised of $\sim 25 \mathrm{nt}$ and two small hairpins extruding from either side of the central stem $20 \mathrm{nt}$ before the loop (Weissenmayer et al., 2011). Many of these sequences were found in other Legionella strains as well, often in the same configuration, which indicates that they are evolutionarily conserved and likely to play a beneficial role. Moreover, both the Lqs system and the homologous sRNA sequences are absent in L. longbeachae. These observations are only suggestive and experimental evidence is needed to link the Lqs quorum sensing system with this group of homologous sRNA sequences. It is noteworthy that deletion of all four Qrr sRNAs was needed to see a phenotype on the quorum sensing system (Lenz et al., 2004). Since only Lpr0001 and Lpr0069 seem to be expressed at good level, it might be informative to generate a double 1 pr0001/lpr0069 mutant and monitor its effect on a population density-related phenotype.

Although the vast majority of base-pairing sRNAs do not encode proteins, there are at least two examples where they do. In E. coli the $\operatorname{sgrS}$ gene encodes a sRA, SgrS, and a small protein, SgrT, that together regulate glucose uptake by different strategies (Wadler and Vanderpool, 2007). In S. aureus, the sRNA RNA III targets virulence factors and functions as a key regulator of virulence, but also encodes a 26 amino acid long hemolysin (Boisset et al., 2007). Therefore, one should keep in mind that sRNAs are not necessarily non-coding. We recently identified two small RNA molecules, LstA and LstB that are predicted to encode small proteins with transmembrane motifs (Faucher et al., 2010). Because small proteins are difficult to predict accurately from genomic sequences, the hunt for small RNA molecules also has the potential benefit of filling the gaps of genomic annotation by also identifying putative small proteins and correcting errors in genome annotation.

\section{THE CsrA/CsrB SYSTEM}

The CsrA protein was first identified in E. coli as a regulator of glycogen biosynthesis (Romeo et al., 1993). CsrA binds to GGA motifs in the 5'UTR of target mRNAs and affects their stability and/or their translation (Romeo, 1998). The sRNAs CsrB and CsrC contain many GGA motifs and can therefore bind multiple CsrA proteins resulting in titration/sequestration of CsrA, thus relieving CsrA effects on the expression of its target mRNAs (Figure 1D). Transcription of CsrB and CsrC is regulated by the BarA/UvrY TCS. Both sRNAs are degraded by a pathway involving RNase E and CsrD, a cyclic di-GMP binding protein (Suzuki et al., 2006).

Legionella pneumophila contains four CsrA homologs, of which one (lpg0781) was identified as able to complement a csrA deletion in E. coli (Fettes et al., 2001). The roles of the other CsrA homologs are currently unknown. In L. pneumophila, CsrA is responsible for the repression of post-exponential traits during exponential growth, including pigmentation, motility, and cell shortening (Fettes et al., 2001; Molofsky and Swanson, 2003; Forsbach-Birk et al., 2004). Moreover, CsrA is required for intracellular growth in both mammalian macrophages and A. castellanii (Molofsky and Swanson, 2003; Forsbach-Birk et al., 2004). Recently, it was shown that CsrA directly repressed the expression of $y l f A / l e g C 7$, $y l f B / \operatorname{leg} C 2$, and $v i p A$, which encode Icm/Dot effectors (Rasis and Segal, 2009b). Regulation of CsrA expression seems to be dependant on PmrA, another well-known virulence regulator (Rasis and Segal, 2009b).
The L. pneumophila genome encodes homologs of the BarA/ UvrY TCS named LetA/LetS. This system was first identified as a positive regulator of flagellin expression (Hammer et al., 2002). Although a let $A$ mutant still replicates in mammalian macrophages, it is defective for replication in A. castellanii (Gal-Mor and Segal, 2003; Lynch et al., 2003). Subsequently, LetA was shown to regulate expression of a number of virulence factors, including Mip, IcmR, IcmT, DotA, and the Icm/Dot effector RalF (Gal-Mor and Segal, 2003; Shi et al., 2006).

Based on these results, the consensus model is that during exponential phase, CsrA represses expression of post-exponential phase genes, either by inhibiting mRNA translation, or by modulating their stability. During post-exponential phase, the LetA/LetS TCS, supposedly by inducing expression of CsrB homologs, inhibits the activity of CsrA, allowing expression of post-exponential traits (pigmentation, cytotoxicity, and motility). Computer predictions of CsrB homologs in several bacterial species identified two candidate CsrB homologs in L. pneumophila, based on the identification of intergenic regions enriched for the GGA motif (Kulkarni et al., 2006). These two sRNAs were named RsmY and RsmZ (Table 1), based on their short size, which more closely resemble the sRNAs involved in the RsmA (CsrA) system of P. aeruginosa (Lapouge et al., 2008). It was shown that: (i) LetA specifically binds upstream of rsm $Y$ and $r s m Z$ and that the LetA/LetS TCS controls their expression; (ii) expression of $r s m Y$ and $r s m Z$ in E. coli results in a similar phenotype as over-expression of $c s r B$ and $c s r C$ and; (iii) RsmY and RsmZ bind CsrA, confirming that RsmY and RsmZ are the missing link in the LetA/S-CsrA regulatory pathway (Hovel-Miner et al., 2009; Rasis and Segal, 2009b; Sahr et al., 2009; Figure 1E). Deletion of either $r s m Y$ or $r s m Z$ has little impact on virulence, but deletion of both strongly impaired replication in both mammalian macrophages and A. castellanii (Sahr et al., 2009). It was also shown that increased expression of $r s m Y$ and $r s m Z$ during post-exponential phase requires RpoS, probably due to the regulation of letS expression by RpoS (Hovel-Miner et al., 2009; Rasis and Segal, 2009b). Reduced expression of CsrA leads to an increase in rpoS expression, which suggests the existence of a positive feedback loop (ForsbachBirk et al., 2004). However, deletion of rsm YZ, which should mimic over-expression of CsrA, also resulted in increased expression of rpoS (Sahr et al., 2009). Therefore, the interplay between LetS, RsmYZ, CsrA, and RpoS remains unclear and will require further investigation (Figure 3).

Interestingly, RpoS and LetA, two major regulators of virulencerelated traits in L. pneumophila positively regulate expression of $h f q$ during exponential growth (McNealy et al., 2005). Whether or not Hfq, in turn, affects RsmY and RsmZ function or stability is currently unknown (Figure 3 ). In P. aeruginosa, Hfq binds to and affects the stability of RsmY (Sonnleitner et al., 2008). Also, the LqsR/LqsS TCS is regulated by the CsrA system, which is similar to what was shown for $V$. cholerae (Lenz et al., 2005; Tiaden et al., 2007; Sahr et al., 2009).

Microarray studies revealed that no genes were significantly affected by the deletion of either letA, letS, or rsmYZ during exponential growth in rich broth, in agreement with the current working model in which CsrA is active during exponential phase and that the LetA/LetS/RsmYZ part of the regulatory cascade is silent (Sahr et al., 2009). However, during the post-exponential phase of 
growth, many genes were negatively affected by deletion of either letA or letS or both $r s m Y Z$, including a number of Icm/Dot effectors (RalF, SidC, SdeA, SdeC, SidF, and SdhB) (Sahr et al., 2009). Independently, it was shown that RsmY and RsmZ relieve the CsrAmediated repression of the expression of $y l f A / l e g C 7, y l f B / l e g C 2$, and $v i p A$ (Rasis and Segal, 2009b). However, expression of flagellar genes was largely RsmYZ independent but negatively affected by deletion of either letA or letS (Sahr et al., 2009). However, since CsrA affects mRNA translation, over-expression of RsmY and RsmZ could result in a stronger phenotype at the protein level. Interestingly several genes positively affected by LetA/S and RsmYZ were predicted to encode GGDEF and/or EAL domains, including lpg0156 (cdgS4) and lpg2132 (cdgs20) (Sahr et al., 2009; Levi et al., 2010), suggesting that there may be crosstalk between the CsrA system and the cyclic di-GMP system (Figure 3) as it was shown in E. coli (Jonas et al., 2008). Interestingly, wild-type bacteria that over-express $c d g s 20$ are defective for intracellular multiplication (Levi et al., 2010).

\section{THE RNA POLYMERASE/6S RNA SYSTEM}

The 6S RNA of E. coli was first identified and sequenced 40 years ago (Hindley, 1967; Brownlee, 1971). However, its function remained elusive until the year 2000 when Wassarman and Storz (2000) showed that 6 S RNA binds to the $\sigma^{70}$ and the $\beta / \beta^{\prime}$ subunits of RNAP and inhibits transcription of the $r s d$ gene from its $\sigma^{70}$-dependant promoter. Later, it was shown that, in laboratory E. coli strains, deletion of the 6S RNA gene, ssrS, renders cells more resistant to high $\mathrm{pH}$ and less able to compete against wild-type bacteria for survival in deep stationary phase (Trotochaud and Wassarman, 2004, 2006).

In bacteria, functional RNAP holoenzyme consists of the core subunits $\beta / \beta^{\prime} \alpha_{2} \omega$, which associate with a $\sigma$ subunit that provides promoter specificity. In E. coli, the $\sigma^{70}$-RNAP holoenzyme $\left(E \sigma^{70}\right)$ is responsible for bulk transcription during exponential phase. During stationary phase, the $\sigma^{s}$ subunit preferentially associates with the $\beta / \beta^{\prime} \alpha_{2} \omega$ subunits of RNAP to allow transcription of stationary phase genes. The general consensus for the role of 6S RNA's regulatory effect is based on its preferential binding to $\mathrm{E} \sigma^{70}$, compared to $\mathrm{E} \sigma^{\mathrm{S}}$, and the observation that binding of 6S RNA to $\mathrm{E} \sigma^{70}$ inhibits its binding to DNA promoters (Figure 1E). Thus, in the presence of $6 \mathrm{~S} \mathrm{RNA}, \mathrm{E} \sigma^{70}$ is sequestered, promoting the formation of other holoenzymes, such as $E \sigma^{\varsigma}$, that are able to activate transcription from their specific promoters (Wassarman, 2007). Later, it was shown that $\sigma^{70}$-dependant promoters negatively affected by the presence of $6 \mathrm{~S}$ RNA contained a weak - 35 element and an extended - 10 element (Cavanagh et al., 2008). Thus, 6S RNA may function as a competitor for the binding of $E \sigma^{70}$ to a specific subset of promoters.

Following bioinformatic prediction of sRNAs in L. pneumophila, one sRNA showed very high expression during the post-exponential phase of growth, similar to E. coli 6S RNA (Wassarman and Storz, 2000). Its predicted structure was highly similar to the published consensus structure of the widely distributed 6S RNA (Barrick et al., 2005; Trotochaud and Wassarman, 2005). All the previously identified conserved features of 6S RNA homologs were present in the L. pneumophila 6S RNA candidate, including: (i) a 22-nt closing stem with two small bulges; (ii) a central bulge composed of $14 \mathrm{nt}$ on the $5^{\prime}$ strand and $13 \mathrm{nt}$ on the $3^{\prime}$ strand of low \%GC content; (iii) two G-C base pairs surrounding the central bulge; and (iv) a terminal loop comprising four small bulges resembling the consensus terminal loop of the $\gamma$-proteobacteria lineage of 6S RNAs. Co-immunoprecipitation studies revealed that the L. pneumophila 6S RNA candidate physically associate with RNAP (Faucher et al., 2010). Therefore, the gene encoding this sRNA was named ssrS in accordance with the published nomenclature recommendations (Barrick et al., 2005).

Deletion of the ssrS gene reduced intracellular growth in human macrophages and in A. castellanii by 10-fold despite no difference in Icm/Dot translocation activity or cytotoxicity (Faucher et al., 2010). Also, the 6S RNA deficient strain was unable to compete against the wild-type strain during intracellular growth but grew equally well in AYE broth. Thus, it seems that in L. pneumophila 6S RNA is important for optimal expression of genes related to intracellular growth (Figure 3). In order to further dissect the effects of 6S RNA on gene expression, microarray analysis was used to monitor global gene expression patterns during the post-exponential phase of growth, when the 6S RNA is most abundant. When the ssrS deletion mutant strain was compared to the wild-type it was observed that L. pneumophila 6S RNA negatively affects expression of six genes and promotes transcription of 127 genes during post-exponential phase of growth, including those encoding: a subset of $\mathrm{Icm} / \mathrm{Dot}$ effectors (VipA, LegC5, SdeC, SdbC), small molecule transporters, DNA repair enzymes as well as genes involved in fatty acid metabolism, amino acid metabolism, and carbohydrate metabolism. This was somewhat in contradiction with the consensus understanding of 6S RNA being mainly an inhibitor of transcription from $\sigma^{70}$-dependant promoters. However, a recent study revealed that $6 \mathrm{~S}$ RNA is also an activator of transcription in $E$. coli, where it negatively affects transcription of 148 genes and positively affects expression of 125 genes (Neusser et al., 2010). In this study, genes affected by $6 \mathrm{~S}$ RNA contain promoters that are specific for a variety of $\sigma$ subunits, including $\sigma^{S}, \sigma^{32}$, and $\sigma^{54}$. Accordingly, 6S RNA seems to bind also to E $\sigma^{s}$, although with much less affinity than for $E \sigma^{70}$ (Gildehaus et al., 2007). Therefore, it seems that 6S RNA regulation is not as clear-cut as first conceived and these results suggest that many variations on a common theme may exist in different bacterial species. Factors that could influence 6S RNA regulation in L. pneumophila include distinctive usage of the different $\sigma$ subunits, strength of the promoters present in the genome and overall regulatory organization.

In E. coli, RNAP can use 6S RNA as a template to generate 14-24 nt long de novo RNA molecules, named pRNA, originating from the central bulge on the 5' strand (Wassarman and Saecker, 2006; Gildehaus et al., 2007). However, transcription from 6S RNA only occurs after a sudden increase in the NTP pool, for example when bacteria in post-exponential phase are diluted with fresh medium. Transcription from 6S RNA leads to the dissociation of 6S RNA from $\mathrm{E}^{70}$, which is then free to transcribe genes again. This also causes destabilization of 6S RNA, due to increased access of nucleases to unbound 6S RNA or recognition of the 6S RNA-pRNA duplex by RNases (Wassarman and Saecker, 2006). Therefore, synthesis of pRNA seems to be a way to "reset" this regulatory system. Synthesis of pRNA probably also occurs in other bacteria as well, including L. pneumophila, but at present direct evidence for this is lacking. 
Some bacterial species contain two or more 6S RNA homologs, such as Bacillus subtilis and Clostridium (Barrick et al., 2005; Trotochaud and Wassarman, 2005). A second 6S RNA homolog, named 6S2 RNA, was recently identified in the L. pneumophila genome (Weissenmayer et al., 2011). Surprisingly, the authors could detect transcription from the opposite strand encoding 6S2 RNA and suggest that its expression is regulated by a cis-acting sRNA. The 6S2 RNA is expressed in E and PE phase at a similar level, but the antisense transcript is only expressed in E phase, which could inhibit 6S2 function during E phase and therefore effectively result in functional 6S2 RNA expression only during PE phase. That would result in a situation similar to the 6S RNA of E. coli and the 6S RNA of L. pneumophila that are only highly expressed in PE phase. The role of 6S2 RNA is currently unknown and it would be interesting to investigate the phenotype of a mutant defective in both 6S RNA and 6S2 RNA.

\section{THE CRISPR IMMUNITY SYSTEM}

The CRISPR loci encode a sRNA-based immunity system against viruses and other invading DNA (Horvath and Barrangou, 2010). It consists of a leader sequence followed by several non-contiguous direct repeats separated by pieces of variable sequences called spacers. The spacer is a sequence of DNA (21-72 bp) originating from invading viral or plasmid DNA that has been integrated in the bacterial genome. Following transcription of the CRISPR loci, the multi-repeat, multi-spacer RNA is processed by CRISPR-associated protein (Cas) into small units consisting of a spacer flanked by two partial repeats, called crRNA. Those crRNA provide specificity to the system by guiding the Cas interference machinery to the invading nucleic acids that match its sequence. Therefore, the spacers are remnants of past viral infections or plasmid invasions and can be viewed as a form of acquired immunity. New spacers can be added at the leader end of the CRISPR loci.

In L. pneumophila, CRISPR loci have been identified in the Lens, Alcoy, and Paris strains, but not in Philadelphia-1 (D'Auria et al., 2010). The Lens strain possess two CRISPR loci, one on the chromosome, the other on a plasmid. The Alcoy and Lens CRISPR systems are almost identical, composed of three Cas genes (cas1, cas3, and csy4) and 55 or 52 repeats, respectively, of 27 bp with one bp difference between the two strains. The Paris locus are not related to the Alcoy/Lens loci and is composed of cas1, cas2, and cas 4 and contains 34 repeats of $37 \mathrm{bp}$. BLAST analysis of the spacer sequences did not identify any homologous sequences in the GenBank database. It is noteworthy that four bacteriophages of L. pneumophila have been identified from environmental water samples, but their sequences are unknown (Lammertyn et al., 2008). There is currently no evidence of any implication of the CRISPR system in regulation of virulence-related traits in L. pneumophila. However, in P. aeruginosa, the CRISPR system is needed for bacteriophage-mediated inhibition of biofilm formation and swarming motility following lysogenic infection with bacteriophage DMS3 (Zegans et al., 2009). This suggests that the combination of lysogenic infection and the presence of an active CRISPR system may have an impact on the regulation of group behavior traits. Whether or not this is relevant in the context of host infection by bacterial pathogens still needs to be determined.

\section{RELIEF OF STALLED RIBOSOMES BY tmRNA}

Stalling of ribosomes on a mRNA occurs when the translation machinery reaches the end of of the transcript without encountering a stop codon. This is a consequence of co-transcriptional translation that occurs in bacteria and the translation of mRNA that are being degraded from the $3^{\prime}$ end. Stalling of the ribosome prevents its release from the mRNA and can cause decay of the active ribosome pool. Moreover, generation of incomplete proteins can be toxic to the cells. Therefore, a system is needed to release the ribosome and target the incomplete protein for degradation. This function is performed by the tmRNA that is universally conserved in the bacterial kingdom (reviewed in Keiler, 2007; Table 1). The name tmRNA comes from the two functions performed by this sRNA. It acts as a tRNA and is charged with alanine and it acts as an mRNA, encoding a short peptide tag, which targets a protein for degradation. The current model of tmRNA-mediated rescue of stalled ribosome includes two proteins: SmpB and EF-Tu. A complex formed from alanyl-tmRNA-SmpB-EF-Tu enters the A-site of the stalled ribosome. The nascent protein is transferred to the alanyl-tmRNA. The complex then moves to the P-site and the ribosome translates the short peptide tag encoded on the tmRNA, resulting in tagging of the protein and release of the mRNA. Deletion of tmRNA usually results in strong phenotypes such as a marked reduction in growth rate and lethality (Keiler, 2007). In the intracellular pathogen Salmonella, deletion of tmRNA or the $\operatorname{smpB}$ gene results in severe reduction in survival capacity and pathogenesis in mouse macrophages (Julio et al., 2000; Ansong et al., 2009). The effect of the deletion of tmRNA in L. pneumophila is currently unknown but $\mathrm{SmpB}$ may be essential for axenic growth since a $\operatorname{smp} B$ deletion mutant could not be constructed (Charpentier et al., 2008).

\section{A NOTE ABOUT 5' AND 3' UNTRANSLATED REGIONS OF mRNA}

In addition to their coding sequences, mRNAs have two distinct regions that can perform regulatory functions: the 5'UTR and the 3'UTR (Gripenland et al., 2010). Both regions can vary greatly in length from only a few, to several hundred bases. Some 5'UTR can adopt different structural states depending on conditions inside cells, including temperature (e.g., thermosensor), $\mathrm{pH}$, and the presence of specific metabolites (Figure 1A). Such 5'UTR are called riboswitches. One of the best-known riboswitches regulates transcription of the $\operatorname{prfA}$ gene, a major virulence regulator of Listeria monocytogenes. At low temperatures, the prfA $5^{\prime} \mathrm{UTR}$ adopts a structural state that masks the RBS and thus prevents translation. In contrast, at $37^{\circ} \mathrm{C}$, the $5^{\prime} \mathrm{UTR}$ structure changes, exposing the RBS and allowing translation of the PrfA protein and expression of virulence determinants (Johansson et al., 2002). No riboswitches have been identified in L. pneumophila as yet. However, temperature is known to affect biofilm formation by L. pneumophila (Piao et al., 2006). Moreover, optimal growth at high and low temperature requires specific stress response proteins: ClpP and RNase R respectively (Charpentier et al., 2008; Li et al., 2010). Therefore, one may speculate that RNA thermosensors could be involved in L. pneumophila gene regulation to promote growth at extreme temperatures and to form biofilms.

The small nucleotide cyclic di-GMP regulates many biological processes in bacteria, including biofilm formation, motility, and virulence (Hengge, 2009). Cyclic di-GMP is produced from 
two guanosine-5'-triphosphate molecules by diguanylate cyclases (DGC, containing a GGDEF domain) and degraded selectively by phosphosdiesterases containing either EAL or HD-GYP domains (Hengge, 2009). Therefore, the quantities and activities of DGC and EAL/HD-GYP enzymes determine the net intracellular concentration of cyclic di-GMP, which may be an integration point for many different signals. Consequently, the mechanism(s) of gene regulation by cyclic di-GMP has been the subject of intense investigation. A new riboswitch class that regulates gene expression by binding to the second messenger cyclic di-GMP was described and found in many different bacterial species (Sudarsan et al., 2008). Recently, our lab provided evidence that the cyclic di-GMP signaling pathway of L. pneumophila is involved in the regulation of intracellular growth and flagellin synthesis (Levi et al., 2010). Given the large number of DGC and EAL/HD-GYP enzymes present in L. pneumophila genome, it is tempting to speculate that an, as yet, unidentified riboswitch may play a role in cyclic di-GMP regulatory pathways in L. pneumophila. However, no riboswitch has been identified in L. pneumophila as yet and it would therefore be interesting to performed a systematic search to identify possible candidate.

In eukaryotes, $3^{\prime}$ UTRs are important for the control of translation (Sonenberg and Hinnebusch, 2009). The importance of 3'UTR for bacterial gene regulation is currently unclear but probably underestimated. Long overlapping 3'UTRs were identified in $L$. monocytogenes and in B. subtilis (Rasmussen et al., 2009; ToledoArana et al., 2009). Such 3'UTRs could affect the stability of convergent genes by a mechanism similar to cis-encoded base-pairing sRNAs (see below). Whole-genome tiling array experiments were used to find transcriptionally active regions in B. subtilis, which identified a group of genes with long ( 200 nt) homologous 3 'UTR (Rasmussen et al., 2009). Structure predictions revealed that those 3'UTR fold into a highly stable Y-shaped double-stranded structure ending with a very short single-stranded tail. The author suggested that such structures could target the mRNA to a location in the cells were the protein is needed (i.e., the membrane) or prevent access of RNAses to the $3^{\prime}$ end of the transcript. Stable structures at the $3^{\prime}$ end of mRNAs block the activities of most $3^{\prime}$-exoribonucleases. RNase $\mathrm{R}$ is able to degrade double-stranded RNA molecules but needs a single-stranded tail of at least $10 \mathrm{nt}$ (Vincent and Deutscher, 2006). In L. pneumophila, six actively transcribed 3'UTRs were identified (Table 1), ranging from 66 to 180 bases (Faucher et al., 2010). Whether or not they are involved in gene regulation requires clarification. Interestingly, the predicted structure of $g l t X-3^{\prime} U T R$ is similar to the Y-shape structure reported in B. subtilis homologous $3^{\prime} \mathrm{UTR}$.

\section{THE NEXT STEP: TARGET IDENTIFICATION AND CHARACTERIZATION OF L. PNEUMOPHILA SRNAS}

Now that a number of actively transcribed sRNAs have been identified in L. pneumophila, further research should focus on the determination of their functions and their specific targets. First of all, it is important to define what a true target is. Essentially, a true target is a mRNA or a protein that physically interacts with the sRNA and whose function, stability or translation is affected by this interaction (Vogel and Wagner, 2007). The inferred targets of cis-encoded base-pairing sRNAs are obvious, they are the mRNA encoded on the complementary strand. However, even in this case, molecular evidence is needed to establish the link between the two molecules and the effect of the sRNA on the target mRNA. For trans-encoded base-pairing sRNAs, there are a priori no indications of what the target might be. As a start, it could be useful to use a bioinformatic approach to generate a list of putative targets that can then be tested experimentally. Target prediction usually relies on the estimation of optimal hybridization scores between sRNA and mRNA targets and often includes the effects of stable secondary structures. Many web servers are available for genome-wide prediction of mRNA targets, including, but not limited to sRNATarget (Cao et al., 2009) and TargetRNA (Tjaden et al., 2006).

Target prediction could also be used in conjunction with experimental genome-wide approaches such as transcriptional profiling. Comparison of the transcription profile of a mutant strain or an over-expresser strain to the wild-type strain can highlight putative targets (Papenfort et al., 2008). One has to keep in mind that any observed effects on transcript expression could be indirect, when, for example, a transcriptional regulator is the true target. Since the effect of some sRNAs can only be seen at the protein level, the effect of a sRNA is not necessarily observable at the steady-state RNA level. Comparison at the proteome level, by 2D gel analysis, could be more informative, but because of detection limitation, poorly expressed proteins are usually missed. Comparison of the sRNA deletion mutant, the over-expresser strain and the wild-type strain by SDS-PAGE and Coomassie staining may be sufficient to suggest a putative target. Then, a protein of interest can be identified by mass spectrometry analysis. The target of the GlmY sRNA, a polycistronic mRNA encoding glmUS, was identified with this strategy (Urban et al., 2007).

A more direct approach to find the mRNA target of transencoded sRNA is to use the sRNA as a bait to fish out the target. In the case of a sRNA that interact with Hfq, the sRNA-Hfq complex can be preloaded into an affinity purification column and incubated with extracted mRNA. After washing, the eluted mRNA are converted to cDNA and identified by sequencing or by microarray analysis. Such method was used to identify the target of the E. coli RydC sRNA, an ATP-binding cassette permease (Antal et al., 2005). Alternatively, a sRNA could be tagged with biotin, bound to streptavidin-coated magnetic beads and incubated with extracted mRNA. Identification of the captured mRNA could be performed as explained above. This method has been used to identify two targets, ompA and $o m p C$ mRNA, of the RseX sRNA of $E$. coli (Douchin et al., 2006).

The identification of protein targets of protein-binding sRNAs is somewhat similar to what was described above for mRNAbinding sRNAs. However, in this case, secondary structures are often very well conserved, which is illustrated by 6S RNA and the CsrB homologs, and therefore structure predictions could serve as a guide. Then proteomic studies could be undertaken or more direct approaches, such as the streptavidin-binding aptamer tag described above could be used (Windbichler et al., 2008). Said et al. (2009) have performed a systematic analysis of the use of different aptamers and configurations to identify protein targets of sRNA. 


\section{CONCLUDING REMARKS}

Increasing evidence points to important and broad implications of sRNAs in the regulation of life cycles, stress responses and virulence properties of several pathogenic bacteria (Papenfort and Vogel, 2010). This is evident in L. pneumophila where three sRNAs, 6S RNA, RsmY, and RsmZ, are already known as major determinants of virulence regulation. However, this is probably only the tip of the iceberg and it is likely that other sRNAs are involved in regulation of virulence and other traits such as biofilm formation and the responses to environmental stresses. In the near future, important goals for characterizing the specific roles of sRNA in L. pneumophila

\section{REFERENCES}

Abu-Zant, A., Asare, R., Graham, J. E., and Abu Kwaik, Y. (2006). Role for RpoS but not RelA of Legionella pneumophila in modulation of phagosome biogenesis and adaptation to the phagosomal microenvironment. Infect. Immun. 74, 3021-3026.

Altman, E., and Segal, G. (2008). The response regulator $\mathrm{CpxR}$ directly regulates expression of several Legionella pneumophila icm/dot components as well as new translocated substrates. $J$. Bacteriol. 190, 1985-1996.

Altuvia, S., Weinstein-Fischer, D., Zhang, A., Postow, L., and Storz, G. (1997). A small, stable RNA induced by oxidative stress: role as a pleiotropic regulator and antimutator. Cell 90, 43-53.

Ansong, C., Yoon, H., Porwollik, S., Mottaz-Brewer, H., Petritis, B. O., Jaitly, N., Adkins, J. N., McClelland, M., Heffron, F., and Smith, R. D. (2009). Global systems-level analysis of Hfq and SmpB deletion mutants in Salmonella: implications for virulence and global protein translation. PLoS ONE 4, e4809. doi: 10.1371/journal. pone.0004809

Antal, M., Bordeau, V., Douchin, V., and Felden, B. (2005). A small bacterial RNA regulates a putative $\mathrm{ABC}$ transporter. J. Biol. Chem. 280, 7901-7908.

Bardill, J. P., Miller, J. L., and Vogel, J. P. (2005). IcmS-dependent translocation of SdeA into macrophages by the Legionella pneumophila type IV secretion system. Mol. Microbiol.56,90-103.

Barrick, J. E., Sudarsan, N., Weinberg, Z., Ruzzo, W.L., and Breaker, R. R. (2005). 6S RNA is a widespread regulator of eubacterial RNA polymerase that resembles an open promoter. $R N A$ 11, 774-784.

Benin, A. L., Benson, R. F., and Besser, R. E. (2002). Trends in legionnaires disease, 1980-1998: declining mortality and new patterns of diagnosis. Clin. Infect. Dis. 35, 1039-1046.

Bohn, C., Rigoulay, C., and Bouloc, P. (2007). No detectable effect of RNAbinding protein $\mathrm{Hfq}$ absence in Staphylococcus aureus. BMCMicrobiol. 7, 10. doi: 10.1186/1471-2180-7-10
Boisset, S., Geissmann, T., Huntzinger, E., Fechter, P., Bendridi, N., Possedko, M., Chevalier, C., Helfer, A. C., Benito, Y., Jacquier, A., Gaspin, C., Vandenesch, F., and Romby, P. (2007). Staphylococcus aureus RNAIII coordinately represses the synthesis of virulence factors and the transcription regulator Rot by an antisense mechanism. Genes Dev. 21, 1353-1366.

Brantl, S. (2007). Regulatory mechanisms employed by cis-encoded antisense RNAs. Curr. Opin. Microbiol.10, 102-109.

Brownlee, G. G. (1971). Sequence of $6 \mathrm{~S}$ RNA of E. coli. Nat. New Biol. 229, 147-149.

Burstein, D., Zusman, T., Degtyar, E., Viner, R., Segal, G., and Pupko, T. (2009). Genome-scale identification of Legionella pneumophila effectors using a machine learning approach. PLoS Pathog. 5, e1000508. doi: 10.1371/journal.ppat.1000508

Cao, Y., Zhao, Y., Cha, L., Ying, X., Wang, L., Shao, N., and Li, W. (2009). sRNATarget: a web server for prediction of bacterial sRNA targets. Bioinformation 3, 364-366.

Cavanagh, A. T., Klocko, A. D., Liu, X., and Wassarman, K. M. (2008). Promoter specificity for 6S RNA regulation of transcription is determined by core promoter sequences and competition for region 4.2 of sigma70. Mol. Microbiol. 67, 1242-1256.

Cazalet, C., Rusniok, C., Bruggemann, H., Zidane, N., Magnier, A., Ma, L., Tichit, M., Jarraud, S., Bouchier, C., Vandenesch, F., Kunst, F., Etienne, J., Glaser, P., and Buchrieser, C. (2004). Evidence in the Legionella pneumophila genome for exploitation of host cell functions and high genome plasticity. Nat. Genet. 36, 1165-1173.

Chao, Y., and Vogel, J. (2010). The role of Hfq in bacterial pathogens. Curr. Opin. Microbiol. 13, 24-33.

Charpentier, X., Faucher, S. P., Kalachikov, S., and Shuman, H. A. (2008). Loss of RNase R induces competence development in Legionella pneumophila. J. Bacteriol. 190, 8126-8136.

Charpentier, X., Kay, E., Schneider, D., and Shuman, H.A. (2011). Antibiotics

biology are the identification of sRNA targets and determining the phenotypes of mutant that are defective in the production of individual and multiple sRNA species.

\section{ACKNOWLEDGMENTS}

This work was supported by PHS award AI064881 to Howard A. Shuman. Sébastien P. Faucher was supported by a post-doctoral fellowship from the National Sciences and Engineering Research Council of Canada (NSERC) and from the Fond de recherche en santé du Québec (FRSQ). We would like to thank three anonymous reviewers for helpful comments and suggestions.

and UV radiation induce competence for natural transformation in Legionella pneumophila. J. Bacteriol. 193, 1114-1121.

Chaulk, S., Smith-Frieday, M. N., Arthur, D. C., Culham, D. E., Edwards, R. A., Soo, P., Frost, L. S., Keates, R. A. B., Glover, J. N. M., and Wood, J. M. (2011). ProQ is an RNA chaperone that controls ProP levels in Escherichia coli. Biochemistry 50, 3095-3106.

Chenna, R., Sugawara, H., Koike, T., Lopez, R., Gibson, T. J., Higgins, D. G., and Thompson, J. D. (2003). Multiple sequence alignment with the Clustal series of programs. Nucleic Acids Res. 31, 3497-3500.

Chien, M., Morozova, I., Shi, S., Sheng, H. Chen, J., Gomez, S. M., Asamani, G. Hill, K., Nuara, J., Feder, M., Rineer, J., Greenberg, J. J., Steshenko, V., Park, S. H., Zhao, B., Teplitskaya, E., Edwards, J. R., Pampou, S., Georghiou, A., Chou, I. C., Iannuccilli, W., Ulz, M. E., Kim, D. H., Geringer-Sameth, A., Goldsberry, C., Morozov, P., Fischer, S. G., Segal, G., Qu,X., Rzhetsky,A.,Zhang, P., Cayanis, E., De Jong, P. J., Ju, J., Kalachikov, S., Shuman, H. A., and Russo, J. J. (2004). The genomic sequence of the accidental pathogen Legionella pneumophila. Science 305, 1966-1968.

Christiansen, J. K., Nielsen, J. S. Ebersbach, T., Valentin-Hansen, P., Sogaard-Andersen, L., and Kallipolitis, B. H. (2006). Identification of small Hfq-binding RNAs in Listeria monocytogenes. RNA 12, 1383-1396.

Cirillo, S. L., Bermudez, L. E., El-Etr, S. H., Duhamel, G. E., and Cirillo, J. D. (2001). Legionella pneumophila entry gene rtxA is involved in virulence. Infect. Immun. 69, 508-517.

Cirillo, S. L., Yan, L., Littman, M., Samrakandi, M. M., and Cirillo, J. D. (2002). Role of the Legionella pneumophila rtxA gene in amoebae. Microbiology 148, 1667-1677.

D’Auria, G., Jimenez-Hernandez, N., Peris-Bondia, F., Moya, A., and Latorre, A. (2010). Legionella pneumophila pangenome reveals strain-specific virulence factors. BMC Genomics 11, 181. doi: 10.1186/1471-2164-11-181 de Felipe, K. S., Pampou, S., Jovanovic, O. S., Pericone, C. D., Ye, S. F., Kalachikov, S., and Shuman, H.A. (2005). Evidence for acquisition of Legionella type IV secretion substrates via interdomain horizontal gene transfer. J. Bacteriol. 187, 7716-7726.

Dietrich, M., Munke, R., Gottschald, M., Ziska, E., Boettcher, J. P., Mollenkopf, H., and Friedrich,A. (2009). The effect of hfq on global gene expression and virulence in Neisseria gonorrhoeae. FEBS J. 276, 5507-5520.

Douchin, V., Bohn, C., and Bouloc, P. (2006). Down-regulation of porins by a small RNA bypasses the essentiality of the regulated intramembrane proteolysis protease RseP in Escherichia coli. J. Biol. Chem. 281, 12253-12259.

Faucher, S. P., Friedlander, G., Livny, J., Margalit, H., and Shuman, H. A. (2010). Legionella pneumophila 6S RNA optimizes intracellular multiplication. Proc. Natl. Acad. Sci. U.S.A. 107, 7533-7538.

Faucher, S. P., Mueller, C. A., and Shuman, H. A. (2011). Legionella pneumophila transcriptome during intracellular multiplication in human macrophages. Front. Microbiol. 2:60. doi: 10.3389/fmicb.2011.00060

Fettes, P. S., Forsbach-Birk, V., Lynch, D., and Marre, R. (2001). Overexpresssion of a Legionella pneumophila homologue of the E. coli regulator csrA affects cell size, flagellation, and pigmentation. Int. J. Med. Microbiol. 291, 353-360.

Figueroa-Bossi, N., Valentini, M., Malleret, L., Fiorini, F., and Bossi, L. (2009). Caught at its own game: regulatory small RNA inactivated by an inducible transcript mimicking its target. Genes Dev. 23, 2004-2015.

Forsbach-Birk, V., McNealy, T., Shi, C., Lynch, D., and Marre, R. (2004). Reduced expression of the global regulator protein CsrA in Legionella pneumophila affects virulenceassociated regulators and growth in Acanthamoeba castellanii. Int. J. Med. Microbiol. 294, 15-25.

Franco, I. S., Shuman, H. A., and Charpentier, X. (2009). The perplexing functions and surprising origins 
of Legionella pneumophila type IV secretion effectors. Cell. Microbiol. 11, 1435-1443.

Fraser, D. W., Tsai, T. R., Orenstein, W., Parkin, W. E., Beecham, H. J., Sharrar, R. G., Harris, J., Mallison, G. F., Martin, S. M., McDade, J. E., Shepard, C. C., and Brachman, P. S. (1977). Legionnaires' disease: description of an epidemic of pneumonia. N. Engl. J. Med. 297, 1189-1197.

Gal-Mor, O., and Segal, G. (2003). The Legionella pneumophila GacA homolog (LetA) is involved in the regulation of $\mathrm{icm}$ virulence genes and is required for intracellular multiplication in Acanthamoeba castellanii. Microb. Pathog. 34, 187-194.

Gildehaus, N., Neusser, T., Wurm, R., and Wagner, R. (2007). Studies on the function of the riboregulator 6S RNA from E. coli: RNA polymerase binding, inhibition of in vitro transcription and synthesis of RNA-directed de novo transcripts. Nucleic Acids Res. 35 , 1885-1896.

Gripenland, J., Netterling, S., Loh, E., Tiensuu, T., Toledo-Arana, A., and Johansson, J. (2010). RNAs: regulators of bacterial virulence. Nat. Rev. Microbiol. 8, 857-866.

Hales, L. M., and Shuman, H. A. (1999). The Legionella pneumophila rpoS gene is required for growth within Acanthamoeba castellanii. J. Bacteriol. 181, 4879-4889.

Hammer, B. K., Tateda, E. S., and Swanson, M. S. (2002). A two-component regulator induces the transmission phenotype of stationary-phase Legionella pneumophila. Mol. Microbiol. 44, 107-118.

Hansen, A. M., and Kaper, J. B. (2009). Hfq affects the expression of the LEE pathogenicity island in enterohaemorrhagic Escherichia coli. Mol. Microbiol. 73, 446-465.

Hengge, R. (2009). Principles of c-diGMP signalling in bacteria. Nat. Rev. Microbiol. 7, 263-273.

Hindley, J. (1967). Fractionation of 32P-labelled ribonucleic acids on polyacrylamide gels and their characterization by fingerprinting. J. Mol. Biol. 30, 125-136.

Horvath, P., and Barrangou, R. (2010). CRISPR/Cas, the immune system of bacteria and archaea. Science 327, 167-170

Hovel-Miner, G., Pampou, S., Faucher, S. P., Clarke, M., Morozova, I., Morozov, P., Russo, J. J., Shuman, H. A., and Kalachikov, S. (2009). SigmaS controls multiple pathways associated with intracellular multiplication of Legionella pneumophila. J. Bacteriol. 191, 2461-2473.

Huang, L., Boyd, D., Amyot, W. M., Hempstead, A. D., Luo, Z. Q.,
O'Connor, T. J., Chen, C., Machner, M., Montminy, T., and Isberg, R. R. (2011). The E block motif is associated with Legionella pneumophila translocated substrates. Cell. Microbiol.13,227-245.

Hubber, A., and Roy, C. R. (2010). Modulation of host cell function by Legionella pneumophilatype IV effectors. Annu. Rev. Cell Dev. Biol. 26, 261-283.

Johansson, J., Mandin, P., Renzoni, A. Chiaruttini, C., Springer, M., and Cossart, P. (2002). An RNA thermosensor controls expression of virulence genes in Listeria monocytogenes. Cell 110, 551-561.

Jonas, K., Edwards, A. N., Simm, R. Romeo, T., Romling, U., and Melefors, O. (2008). The RNA binding protein CsrA controls cyclic di-GMP metabolism by directly regulating the expression of GGDEF proteins. $\mathrm{Mol}$. Microbiol. 70, 236-257.

Jousselin, A., Metzinger, L., and Felden, B. (2009). On the facultative requirement of the bacterial RNA chaperone, Hfq. Trends Microbiol. 17, 399-405.

Julio, S. M., Heithoff, D. M., and Mahan, M. J. (2000). ssrA (tmRNA) plays a role in Salmonella enterica serovar Typhimurium pathogenesis. J. Bacteriol. 182, 1558-1563.

Keiler, K. C. (2007). Physiology of tmRNA what gets tagged and why? Curr. Opin. Microbiol. 10, 169-175.

Kulkarni, P. R., Cui, X., Williams, J. W., Stevens, A. M., and Kulkarni, R. V. (2006). Prediction of CsrA-regulating small RNAs in bacteria and their experimental verification in Vibrio fischeri. Nucleic Acids Res. 34, 3361-3369.

Lammertyn, E., Vande Voorde, J., Meyen, E. Maes, L., Mast, J., and Anné, J. (2008). Evidence for the presence of Legionella Bacteriophages in environmental water samples. Microb. Ecol. 56, 191-197.

Lapouge, K., Schubert, M., Allain, F. H. and Haas, D. (2008). Gac/Rsm signal transduction pathway of gammaproteobacteria: from $\mathrm{RNA}$ recognition to regulation of social behaviour. $\mathrm{Mol}$. Microbiol. 67, 241-253.

Lenz, D. H., Miller, M. B., Zhu, J., Kulkarni, R. V., and Bassler, B. L. (2005). CsrA and three redundant small RNAs regulate quorum sensing in Vibrio cholerae. Mol. Microbiol. 58, 1186-1202.

Lenz, D. H., Mok, K. C., Lilley, B. N., Kulkarni, R. V., Wingreen, N. S., and Bassler, B. L. (2004). The small RNA chaperone Hfq and multiple small RNAs control quorum sensing in Vibrio harveyi and Vibrio cholerae. Cell 118, 69-82.

Levi, A., Folcher, M., Jenal, U., and Shuman, H. A. (2011). Cyclic diguanylate signaling proteins control intracellular growth of Legionella pneumophila. MBio. 2, doi: 10.1128/ mBio.00316-10
Li, X. H., Zeng, Y. L., Gao, Y., Zheng, X. C., Zhang, Q. F., Zhou, S. N., and $\mathrm{Lu}, \mathrm{Y}$. J. (2010). The ClpP protease homologue is required for the transmission traits and cell division of the pathogen Legionella pneu mophila. BMC Microbiol. 10, 54. doi 10.1186/1471-2180-10-54

Liu, Y., Wu, N., Dong, J., Gao, Y., Zhang, X., $\mathrm{Mu}, \mathrm{C}$., Shao, N., and Yang, G. (2010). $\mathrm{Hfq}$ is a global regulator that controls the pathogenicity of Staphylococcus aureus. PLoS ONE 5, e13069. doi: 10.1371/journal.pone.0013069

Lynch, D., Fieser, N., Gloggler, K., Forsbach-Birk, V., and Marre, R. (2003). The response regulator LetA regulates the stationary-phase stress response in Legionella pneumophila and is required for efficient infection of Acanthamoeba castellanii. FEMS Microbiol. Lett. 219, 241-248.

Mashburn-Warren, L. M., and Whiteley, M. (2006). Special delivery: vesicle trafficking in prokaryotes. $\mathrm{Mol}$. Microbiol. 61, 839-846.

McNealy, T. L., Forsbach-Birk, V., Shi, C. and Marre, R. (2005). The Hfq homolog in Legionella pneumophila demonstrates regulation by LetA and RpoS and interacts with the global regulator CsrA. J. Bacteriol. 187, 1527-1532.

Molofsky, A. B., and Swanson, M. S. (2003). Legionella pneumophila CsrA is a pivotal repressor of transmission traits and activator of replication. Mol. Microbiol. 50, 445-461.

Molofsky, A. B., and Swanson, M. S. (2004). Differentiate to thrive: lessons from the Legionella pneumophila life cycle. Mol. Microbiol. 53, 29-40.

Neusser, T., Polen, T., Geissen, R., and Wagner, R. (2010). Depletion of the non-coding regulatory 6S RNA in E. coli causes a surprising reduction in the expression of the translation machinery. BMC Genomics 11, 165. doi: 10.1186/1471-2164-11-165

Ng, V., Tang, P., Jamieson, F., Drews, S. J. Brown, S., Low, D. E., Johnson, C. C., and Fisman,D.N.(2008). Going with the flow: legionellosis riskin Toronto, Canada is strongly associated with local watershed hydrology. Ecohealth 5, 482-490.

Overgaard, M., Johansen, J., MollerJensen, J., and Valentin-Hansen, P. (2009). Switching off small RNA regulation with trap-mRNA. $\mathrm{Mol}$. Microbiol. 73, 790-800.

Papenfort, K., Pfeiffer, V., Lucchini, S. Sonawane, A., Hinton, J. C., and Vogel, J. (2008). Systematic deletion of Salmonella small RNA genes identifies CyaR, a conserved CRP-dependent riboregulator of OmpX synthesis. Mol Microbiol. 68, 890-906.

Papenfort, K., and Vogel, J. (2010) Regulatory RNA in bacterial pathogens. Cell Host Microbe 8, 116-127.
Pfeiffer, V., Sittka, A., Tomer, R., Tedin, K., Brinkmann, V., and Vogel, J. (2007). A small non-coding RNA of the invasion gene island (SPI-1) represses outer membrane protein synthesis from the Salmonella core genome. Mol. Microbiol. 66, 1174-1191.

Piao, Z., Sze, C. C., Barysheva, O., Iida, K., and Yoshida, S. (2006). Temperatureregulated formation of mycelial matlike biofilms by Legionella pneumophila. Appl.Environ. Microbiol.72, 1613-1622.

Ramos, C. G., Sousa, S. A., Grilo, A. M., Feliciano, J. R., and Leitao, J. H. (2011). The second RNA chaperone Hfq2, is also required for survival to stress and the full virulence of Burkholderio cenocepacia J2315. J. Bacteriol. 193, 1515-1526.

Rasis, M., and Segal, G. (2009a). The LetA-RsmYZ-CsrA regulatory cascade, together with RpoS and PmrA, post-transcriptionally regulates stationary phase activation of Legionella pneumophila Icm/Dot effectors. Mol. Microbiol. 72, 995-1010.

Rasis, M., and Segal, G. (2009b). The LetA-RsmYZ-CsrA regulatory cascade, together with RpoS and PmrA, post-transcriptionally regulates stationary phase activation of Legionella pneumophila Icm/Dot effectors. Mol. Microbiol. 72, 995-1010.

Rasmussen, S., Nielsen, H. B., and Jarmer, H. (2009). The transcriptionally active regions in the genome of Bacillus subtilis. Mol. Microbiol. 73, 1043-1057.

Romeo, T. (1998). Global regulation by the small RNA-binding protein CsrA and the non-coding RNA molecule CsrB. Mol. Microbiol. 29, 1321-1330.

Romeo, T., Gong, M., Liu, M. Y., and Brun-Zinkernagel, A. M. (1993). Identification and molecular characterization of csrA, a pleiotropic gene from Escherichia coli that affects glycogen biosynthesis, gluconeogenesis, cell size, and surface properties. $J$. Bacteriol. 175, 4744-4755.

Roy, C. R., and Tilney, L. G. (2002). The road less traveled: transport of Legionella to the endoplasmic reticulum. J. Cell Biol. 158, 415-419.

Sahr, T., Bruggemann, H., Jules, M., Lomma, M., Albert-Weissenberger, C., Cazalet, C., and Buchrieser, C. (2009). Two small ncRNAs jointly govern virulence and transmission in Legionella pneumophila. Mol. Microbiol. 72, 741-762.

Said, N., Rieder, R., Hurwitz, R., Deckert, J., Urlaub, H., and Vogel, J. (2009). In vivo expression and purification of aptamer-tagged small RNA regulators. Nucleic Acids Res. 37, e133.

Segal, G., Purcell, M., and Shuman, H. A. (1998). Host cell killing and bacterial conjugation require overlapping sets of genes within a $22-\mathrm{kb}$ region of the 
Legionella pneumophilagenome. Proc. Natl. Acad. Sci. U.S.A. 95, 1669-1674.

Segal, G., Russo, J. J., and Shuman, H. A. (1999). Relationships between a new type IV secretion system and the icm/ dot virulence system of Legionella pneumophila. Mol. Microbiol. 34, 799-809.

Segal, G., and Shuman, H.A. (1998). How is the intracellular fate of the Legionella pneumophila phagosome determined? Trends Microbiol. 6, 253-255.

Sexton, J. A., and Vogel, J. P. (2004). Regulation of hypercompetence in Legionella pneumophila. J. Bacteriol. 186, 3814-3825.

Shakhnovich, E. A., Davis, B. M., and Waldor, M. K. (2009). Hfq negatively regulates type III secretion in EHEC and several other pathogens. Mol. Microbiol. 74, 347-363.

Sharma, C. M., Hoffmann, S., Darfeuille, F., Reignier, J., Findeiss, S., Sittka, A., Chabas, S., Reiche, K., Hackermuller, J., Reinhardt, R., Stadler, P. F., and Vogel, J. (2010). The primary transcriptome of the major human pathogen Helicobacter pylori. Nature 464, 250-255.

Shi, C., Forsbach-Birk, V., Marre, R., and McNealy, T. L. (2006). The Legionella pneumophilaglobal regulatory protein LetA affects DotA and Mip. Int. J. Med. Microbiol. 296, 15-24.

Shin, S., and Roy, C. R. (2008). Host cell processes that influence the intracellular survival of Legionella pneumophila. Cell. Microbiol. 10, 1209-1220.

Sittka, A., Lucchini, S., Papenfort, K., Sharma, C. M., Rolle, K., Binnewies, T. T., Hinton, J. C., and Vogel, J. (2008). Deep sequencing analysis of small noncoding RNA and mRNA targets of the global post-transcriptional regulator, Hfq. PLoS Genet. 4, e1000163. doi: 10.1371/journal.pgen.1000163

Sittka, A., Pfeiffer, V., Tedin, K., and Vogel, J. (2007). The RNA chaperone Hfq is essential for the virulence of Salmonella typhimurium. Mol. Microbiol. 63, 193-217.

Sonenberg, N., and Hinnebusch, A. G. (2009). Regulation of translation initiation in eukaryotes: mechanisms and biological targets. Cell 136, 731-745.

Song, T., Mika, F., Lindmark, B., Liu, Z., Schild, S., Bishop, A., Zhu, J., Camilli, A., Johansson, J., Vogel, J., and Wai, S. N. (2008). A new Vibrio cholerae sRNA modulates colonization and affects release of outer membrane vesicles. Mol. Microbiol. 70, 100-111.

Sonnleitner, E., Schuster, M., SorgerDomenigg, T., Greenberg, E. P., and Blasi, U. (2006). Hfq-dependent alterations of the transcriptome profile and effects on quorum sensing in Pseudomonas aeruginosa. Mol. Microbiol. 59, 1542-1558.

Sonnleitner, E., Sorger-Domenigg, T., Madej, M.J., Findeiss, S., Hackermuller,
J., Huttenhofer, A., Stadler, P. F., Blasi, U., and Moll, I. (2008). Detection of small RNAs in Pseudomonas aeruginosa by RNomics and structure-based bioinformatic tools. Microbiology 154, 3175-3187.

Spirig, T., Tiaden, A., Kiefer, P., Buchrieser, C., Vorholt, J. A., and Hilbi, H. (2008). The Legionella autoinducer synthase LqsA produces an alpha-hydroxyketone signaling molecule. J. Biol. Chem. 283, 18113-18123.

Stone, B. J., and Kwaik, Y. A. (1999). Natural competence for DNA transformation by Legionella pneumophila and its association with expression of type IV pili. J. Bacteriol. 181, 1395-1402.

Sudarsan, N., Lee, E. R., Weinberg, Z., Moy, R. H., Kim, J. N., Link, K. H., and Breaker, R. R. (2008). Riboswitches in eubacteria sense the second messenger cyclic di-GMP. Science 321, 411-413.

Suzuki, K., Babitzke, P., Kushner, S. R., and Romeo, T. (2006). Identification of a novel regulatory protein (CsrD) that targets the global regulatory RNAs CsrB and CsrC for degradation by RNase E. Genes Dev. 20, 2605-2617.

Tiaden, A., Spirig, T., Sahr, T., Walti, M.A., Boucke, K., Buchrieser, C., and Hilbi, H. (2010). The autoinducer synthase LqsA and putative sensor kinase LqsS regulate phagocyte interactions, extracellular filaments and a genomic island of Legionella pneumophila. Environ. Microbiol. 12, 1243-1259.

Tiaden, A., Spirig, T., Weber, S. S., Bruggemann, H., Bosshard, R., Buchrieser, C., and Hilbi, H. (2007). The Legionella pneumophila response regulator LqsR promotes host cell interactions as an element of the virulence regulatory network controlled by RpoS and LetA. Cell. Microbiol. 9, 2903-2920.

Tjaden, B., Goodwin, S. S., Opdyke, J. A., Guillier, M., Fu, D. X., Gottesman, S., and Storz, G. (2006). Target prediction for small, noncoding RNAs in bacteria. Nucleic Acids Res. 34, 2791-2802.

Toledo-Arana, A., Dussurget, O., Nikitas, G., Sesto, N., Guet-Revillet, H., Balestrino, D., Loh, E., Gripenland, J., Tiensuu, T., Vaitkevicius, K., Barthelemy, M., Vergassola, M., Nahori, M.A., Soubigou, G., Regnault, B., Coppee, J.Y., Lecuit, M., Johansson, J., and Cossart, P. (2009). The Listeria transcriptional landscape from saprophytism to virulence. Nature 459, 950-956.

Trotochaud, A. E., and Wassarman, K. M. (2004). 6S RNA function enhances long-term cell survival. J. Bacteriol. 186, 4978-4985.

Trotochaud, A. E., and Wassarman, K. M. (2005). A highly conserved 6S RNA structure is required for regulation of transcription. Nat. Struct. Mol. Biol. 12, 313-319.

Trotochaud, A. E., and Wassarman, K. M. (2006). 6S RNA regulation of pspF transcription leads to altered cell survival at high pH. J. Bacteriol. 188, 3936-3943.

Urban, J. H., Papenfort, K., Thomsen, J., Schmitz, R. A., and Vogel, J. (2007). A conserved small RNA promotes discoordinate expression of the glmUS operon mRNA to activate GlmS synthesis. J. Mol. Biol. 373, 521-528.

Vecerek, B., Moll, I., and Blasi, U. (2007) Control of Fur synthesis by the noncoding RNA RyhB and iron-responsive decoding. EMBO J. 26, 965-975.

Vincent, H. A., and Deutscher, M. P. (2006). Substrate recognition and catalysis by the exoribonuclease RNase R. J. Biol. Chem. 281, 29769-29775.

Vogel, J. (2009).A rough guide to the noncoding RNA world of Salmonella. Mol. Microbiol. 71, 1-11.

Vogel, J., Bartels, V., Tang, T. H., Churakov, G., Slagter-Jager, J. G., Huttenhofer, A., and Wagner, E. G. (2003). RNomics in Escherichia coli detects new sRNA species and indicates parallel transcriptional output in bacteria. Nucleic Acids Res. 31, 6435-6443.

Vogel, J., and Wagner, E. G. (2007) Target identification of small noncoding RNAs in bacteria. Curr. Opin. Microbiol. 10, 262-270.

Vogel, J.P.,Andrews, H. L., Wong, S. K., and Isberg, R. R. (1998). Conjugative transfer by the virulence system of Legionella pneumophila. Science 279, 873-876.

von Baum, H., Ewig, S., Marre, R., Suttorp N., Gonschior, S., Welte, T., and Luck, C. (2008). Community-acquired Legionella pneumonia: new insights from the German competence network for community acquired pneumonia. Clin. Infect. Dis. 46, 1356-1364.

Wadler, C. S., and Vanderpool, C. K. (2007). A dual function for a bacterial small RNA: SgrS performs base pairing-dependent regulation and encodes a functional polypeptide. Proc. Natl. Acad. Sci. U.S.A. 104, 20454-20459.

Wassarman, K.M. (2007).6S RNA: a regulator of transcription. Mol. Microbiol. 65, 1425-1431.

Wassarman, K. M., and Saecker, R. M. (2006). Synthesis-mediated release of a small RNA inhibitor of RNA polymerase. Science 314, 1601-1603.

Wassarman, K. M., and Storz, G. (2000).6S RNA regulates E. coli RNA polymerase activity. Cell 101, 613-623.

Waters, L. S., and Storz, G. (2009). Regulatory RNAs in bacteria. Cell 136, 615-628.

Weissenmayer, B. A., Prendergast, J. G. D., Lohan, A. J., and Loftus, B. J. (2011). Sequencing Illustrates the transcriptional response of Legionella pneumophila during infection and identifies seventy novel small noncoding RNAs. PLoS ONE 6, e17570. doi: 10.1371/journal.pone.0017570

Windbichler, N., von Pelchrzim, F., Mayer, O., Csaszar, E., and Schroeder, R. (2008). Isolation of small RNAbinding proteins from $E$. coli: evidence for frequent interaction of RNAs with RNA polymerase. RNA Biol. 5, 30-40. Yamamoto, S., Izumiya, H., Mitobe, J., Morita, M., Arakawa, E., Ohnishi, M., and Watanabe, H. (2011). Identification of a chitin-induced small RNA that regulates the translation of the tfoXVC gene, encoding a positive regulator of natural competence in Vibrio cholerae. J. Bacteriol. 193, 1953-1965.

Zegans, M. E., Wagner, J. C., Cady, K. C., Murphy, D. M., Hammond, J. H., and O'Toole, G. A. (2009). Interaction between bacteriophage DMS3 and host CRISPR region inhibits group behaviors of Pseudomonas aeruginosa. J. Bacteriol. 191, 210-219.

Zhang, A., Wassarman, K. M., Rosenow, C., Tjaden, B. C., Storz, G., and Gottesman, S. (2003). Global analysis of small RNA and mRNA targets of Hfq. Mol. Microbiol. 50, 1111-1124.

Zhu, W., Banga, S., Tan, Y., Zheng, C., Stephenson, R., Gately, J., and Luo, Z. Q. (2011).Comprehensive identification of protein substrates of the Dot/ Icm type IV transporter of Legionella pneumophila. PLoS ONE 6, e17638. doi: 10.1371/journal.pone.0017638

Zusman, T., Aloni, G., Halperin, E., Kotzer, H., Degtyar, E., Feldman, M., and Segal, G. (2007). The response regulator PmrA is a major regulator of the $\mathrm{icm} /$ dot type IV secretion system in Legionella pneumophila and Coxiella burnetii.Mol.Microbiol.63, 1508-1523.

Conflict of Interest Statement: The authors declare that the research was conducted in the absence of any commercial or financial relationships that could be construed as a potential conflict of interest.

Received: 15 January 2011; paper pending published: 01 February 2011; accepted: 19 April 2011; published online: 06 May 2011. Citation: Faucher SP and Shuman $H A$ (2011) Small regulatory RNA and Legionella pneumophila. Front. Microbio. 2:98. doi: 10.3389/fmicb.2011.00098

This article was submitted to Frontiers in Cellular and Infection Microbiology, a specialty of Frontiers in Microbiology. Copyright (C) 2011 Faucher and Shuman. This is an open-access article subject to a non-exclusive license between the authors and Frontiers Media SA, which permits use, distribution and reproduction in other forums, provided the original authors and source are credited and other Frontiers conditions are complied with. 